\title{
A Finite Element Formulation and Nonlocal Theory for the Static and Free Vibration Analysis of the Sandwich Functionally Graded Nanoplates Resting on Elastic Foundation
}

\author{
Van-Ke Tran, ${ }^{1}$ Thanh-Trung Tran, ${ }^{1}$ Minh-Van Phung, ${ }^{1}$ Quoc-Hoa Pham $\mathbb{D}^{2,3}$ \\ and Trung Nguyen-Thoi ${ }^{2,3}$ \\ ${ }^{1}$ Faculty of Mechanical Engineering, Le Quy Don Technical University, Hanoi, Vietnam \\ ${ }^{2}$ Division of Computational Mathematics and Engineering, Institute for Computational Science, Ton Duc Thang University, \\ Ho Chi Minh City, Vietnam \\ ${ }^{3}$ Faculty of Civil Engineering, Ton Duc Thang University, Ho Chi Minh City, Vietnam
}

Correspondence should be addressed to Quoc-Hoa Pham; phamquochoa@tdtu.edu.vn

Received 12 February 2020; Revised 10 July 2020; Accepted 20 August 2020; Published 11 September 2020

Academic Editor: Domenico Acierno

Copyright (c) 2020 Van-Ke Tran et al. This is an open access article distributed under the Creative Commons Attribution License, which permits unrestricted use, distribution, and reproduction in any medium, provided the original work is properly cited.

\begin{abstract}
This article presents a finite element method (FEM) integrated with the nonlocal theory for analysis of the static bending and free vibration of the sandwich functionally graded (FG) nanoplates resting on the elastic foundation (EF). Material properties of nanoplates are assumed to vary through thickness following two types (Type A with homogeneous core and FG material for upper and lower layers and Type B with FG material core and homogeneous materials for upper and lower layers). In this study, the formulation of the four-node quadrilateral element based on the mixed interpolation of tensorial components (MITC4) is used to avoid "the shear-locking" problem. On the basis of Hamilton's principle and the nonlocal theory, the governing equations for the sandwich FG nanoplates are derived. The results of the proposed model are compared with published works to verify the accuracy and reliability. Furthermore, the effects of geometric parameters and material properties on the static and free vibration behaviors of nanoplates are investigated in detail.
\end{abstract}

\section{Introduction}

Nowadays, with the sophisticated development of technology, the investigation of nanostructures has been widely concerned by scientists in the world. However, the studies show that conventional computational theory for millimeter-sized structures is less accurate for nanometer-sized structures. So, in order to improve the accuracy for the analysis of nanostructures, many theories have been proposed such as the modified couple stress theory [1], the strain gradient theory $[2]$, and the nonlocal theory $[3,4]$. Among these theories, the nonlocal theory $[3,4]$ has been used popularly in the literature due to simplicity and high accuracy. Li et al. [5] developed a new nonlocal model to solve the static and dynamic problems for circular elastic nanosolids. Ansari et al. [6] used the nonlocal theory to analyze the free vibration of single- layered graphene plates. Arash and Wang [7] discussed about the nonlocal elastic theory in modeling carbon nanotubes and graphene. Asemi and Farajpour [8] studied thermomechanical vibration of graphene plates including surface effects by decoupling the nonlocal elasticity equations. Jalali et al. [9] used molecular dynamics combined with nonlocal elasticity approaches to investigate the effect of out-of-plane defects on vibration analysis of graphene. In addition, the nonlocal theory was also used in Refs. [10-20] to investigate the various performances of nanoplates. Recently, more attention has focused on the analysis of FG nanoplates considering the small-scale effects. Natarajan et al. [21] examined the vibration behavior of FG nanoplates by using the MoriTanaka homogenization scheme. Jung and Han [22] used the Navier solution to investigate the bending and free vibration responses of the Sigmoid FGM nanoplates. Nami et al. 
[23] studied the thermal buckling of FG nanoplates by using the third-order shear deformation theory (TSDT) combined with nonlocal elasticity. Hashemi et al. [24] analyzed the free vibration of moderately thick circular/annular FG Mindlin plates via nonlocal elasticity. Salehipour et al. [25] developed the analytical solution for free vibration analysis of the FG micro/nanoplates using the three-dimensional theory of elasticity accounting small-scale effect. Salehipour et al. [26] expanded the modified nonlocal elasticity for examining the natural frequency of the FG micro/nanoplates. Ansari et al. [27] analyzed the bending and free vibration responses of the FG nanoplates by using Eringen's nonlocal theory.

In recent years, the application of FG materials has been widely spread in nanoscale devices such as thin films [28, 29] or fully released nanoelectromechanical systems (NEMS) [30] due to their excellent performance. Therefore, investigating various behaviors of the FG material nanostructures has attracted the attention of many researchers. Simsek [31] studied free longitudinal vibration of axially FG tapered nanorods using nonlocal effects. Simsek and Yurtcu [32] analyzed the bending and buckling of FG nanobeams based on the nonlocal Timoshenko beam theory. Natarajan et al. [33] considered the free flexural vibration of FG nanoplates. Nazemnezhad and Hashemi [34] analyzed the nonlinear free vibration of FG nanobeams, and Hashemi et al. $[35,36]$ investigated the nonlinear free vibration of piezoelectric FG nanobeams using nonlocal elasticity. In the above-mentioned studies, most of them used the analytical solutions to analyze the behavior of FG nanoplates. However, the analytical solutions are limited or even impossible when the geometry, boundary conditions, or types of load become more complicated. As an alternative, numerical methods have been proposed to fill in the gaps of these problems.

In the case of the nanostructures resting on the elastic foundation (EF), some typical works can be mentioned here as follows. Wang and Li [37] investigated the static bending behavior of the nanoplates embedded in an EF. Narendar and Gopalakrishnan [38] studied the wave dispersion of a single-layered graphene sheet embedded in an elastic polymer matrix. Pouresmaeeli et al. [39] investigated the vibration behaviors of nanoplates embedded in a viscoelastic medium. Zenkour and Sobhy [40] considered thermal buckling of nanoplates on the EF by using the sinusoidal shear deformation theory. Panyatong et al. [41] combined the nonlocal theory and the surface stress to investigate the bending behavior of nanoplates embedded in an elastic medium.

It is well known that the classical plate theories are limited only for thin plates. Therefore, many studies in recent years have developed Reissner-Mindlin plate theories to investigate both thick and thin plates. However, the shearlocking phenomenon in these theories can arise when the thickness of plates becomes very small. To eliminate the shear-locking phenomenon, many improved techniques have been introduced such as the reduced integration method [42], discrete shear gap method (DSG) [43], assumed natural strains technique (ANS) [44], and mixed interpola- tion of tensorial components (MITC) [45-48]. Among these methods, the four-node quadrilateral element using MITC technique, which gives the so-called MITC4 element, has attracted the interest of many scientists around the world due to the simplicity of formulation and reasonable computational cost.

Based on the best of authors' knowledge, the static and free vibration analysis of sandwich FG nanoplates resting on the elastic foundation using the MITC4 element integrated with the nonlocal theory has not been published yet. This motivates us to develop the MITC4 element integrated with the nonlocal theory to accurately describe the stress-deformation and displacement of the FG nanoplates resting on the elastic foundation in this work. The accuracy and reliability of the proposed method are verified by comparing its numerical results with those available in the literature. Furthermore, the effects of geometric parameters and material properties on the bending and free vibration responses of sandwich FG nanoplates are also investigated in detail.

\section{Theoretical Formulation}

2.1. FG Sandwich Nanoplates. In this study, we consider a sandwich FG nanoplate has length $a$, width $b$, and thickness $h$ which rests on the Winkler foundation. The properties of FG materials of the nanoplate are given by power-law exponent function as follows:

$$
P(z)=P_{m}+\left(P_{c}-P_{m}\right) V_{c},
$$

where $P$ mentions the effective material property such as the modulus of elasticity $E$, mass density $\rho$, and Poisson's ratio $v$; the characters $m$ and $c$ are the metal and ceramic constituents, respectively; $n$ is the power-law index; and $V_{c}$ is the volume fraction of the material. In this study, two types of the FG sandwich nanoplates are considered as shown in Figure 1.

2.1.1. Type A: Sandwich Nanoplates with FG Skins-Homogeneous Core. The bottom and top surfaces are composed of the FG materials, while the core is ceramic (Figure 1(a)), and $V_{c}$ is given by the following formula:

$$
\begin{cases}V_{c}=\left(\frac{z+h_{2}}{h_{1}+h / 2}\right)^{n}, z \in\left[-\frac{h}{2}, h_{1}\right] & \text { bottom surface } \\ V_{c}=1, z \in\left[h_{1}, h_{2}\right] & \text { core } \\ V_{c}=\left(\frac{z-h_{2}}{h / 2-h_{2}}\right)^{n}, z \in\left[h_{2}, \frac{h}{2}\right] & \text { top surface. }\end{cases}
$$

2.1.2. Type B: Sandwich Nanoplates with Homogeneous Skins-FG Core. In this type, the bottom and top surfaces are metal and ceramic, respectively, while the core is 


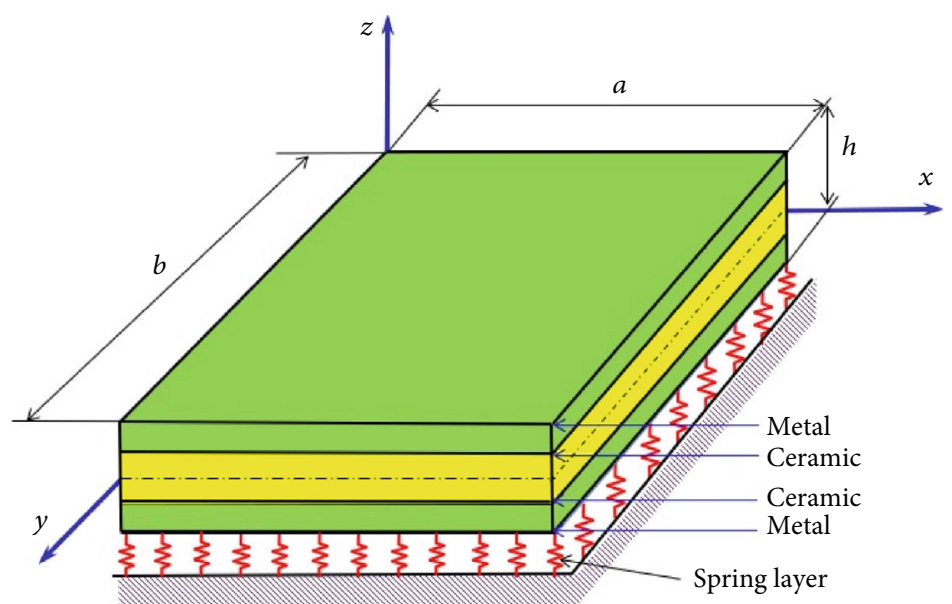

(a)

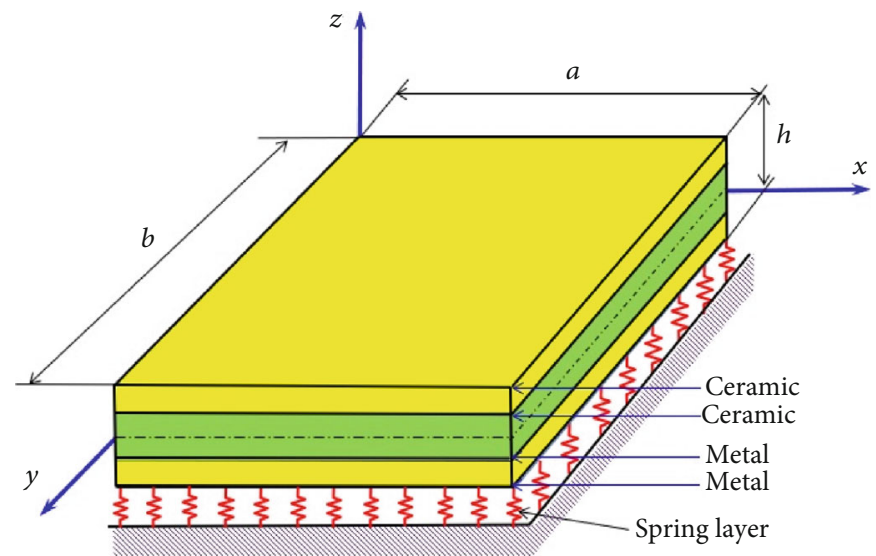

(b)

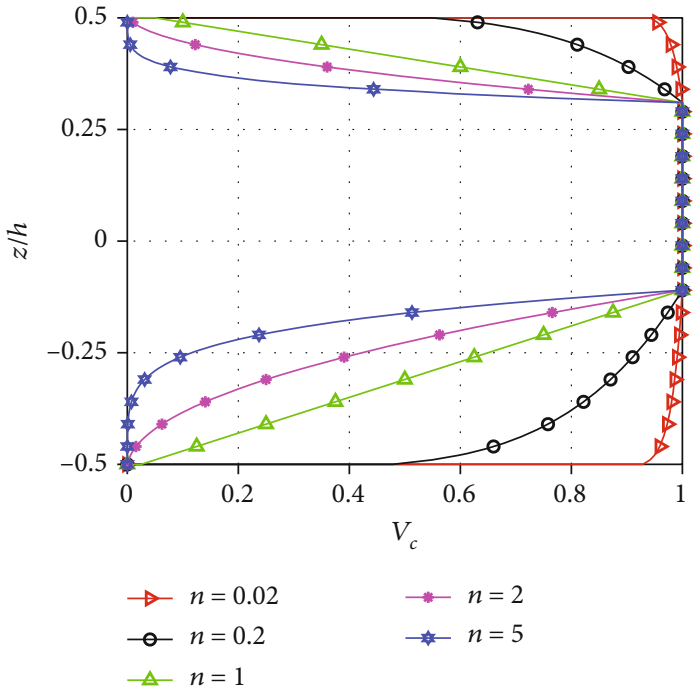

(c)

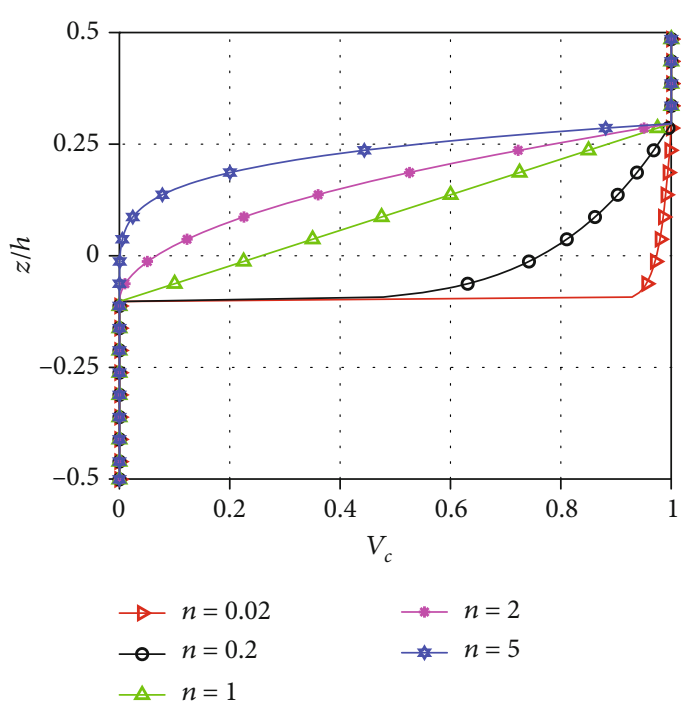

(d)

FigURE 1: Sandwich FG nanoplates rest on EF (a, b) and influence of the power-law index in 2-2-1 sandwich FG nanoplates (c, d). (a, c) Type A. (b, d) Type B. 
composed of the FG materials as shown in Figure 1(b). $V_{c}$ is given by the following formula:

$$
\begin{cases}V_{c}=0, z \in\left[-\frac{h}{2}, h_{1}\right] & \text { bottom skin } \\ V_{c}=\left(\frac{z-h_{1}}{h_{2}-h_{1}}\right)^{n}, z \in\left[h_{1}, h_{2}\right] & \text { core } \\ V_{c}=1, z \in\left[h_{2}, \frac{h}{2}\right] & \text { top skin. }\end{cases}
$$

2.2. Plate Equations Based on Nonlocal Elasticity. Based on the nonlocal theory [1], the constitutive relation of a Hookean solid is determined by

$$
\boldsymbol{\sigma}-\mu \nabla^{2} \boldsymbol{\sigma}=\boldsymbol{Q} ; \mu=\left(e_{0} l\right)^{2},
$$

where $\nabla^{2}=\left(\partial^{2} / \partial x^{2}\right)+\left(\partial^{2} / \partial y^{2}\right) ; \mu$ is the small-scale effect factor or nonlocal factor; $l$ is an internal characteristic length, and $e_{0}$ is a constant; and $\mathbf{Q}$ is the stress tensor at a point which is calculated by the local theory. When $l$ equals zero, the nonlocal continuum theory degenerates into classical elasticity theories. Stress tensor $\boldsymbol{Q}$ is determined by

$$
Q=D \varepsilon,
$$

where

$$
\varepsilon=\left\{\begin{array}{c}
\varepsilon_{x x} \\
\varepsilon_{y y} \\
\varepsilon_{x y} \\
\varepsilon_{x z} \\
\varepsilon_{y z}
\end{array}\right\} ; \sigma=\left\{\begin{array}{c}
\sigma_{x x} \\
\sigma_{y y} \\
\sigma_{x y} \\
\sigma_{x z} \\
\sigma_{y z}
\end{array}\right\}
$$

$\boldsymbol{D}=\left[C_{i j k l}\right]=\left[\begin{array}{ccccc}C_{11} & C_{12} & 0 & 0 & 0 \\ & C_{22} & 0 & 0 & 0 \\ & & C_{66} & 0 & 0 \\ & & & C_{44} & 0 \\ \text { sym } & & & & C_{55}\end{array}\right]=\left[\begin{array}{cc}\boldsymbol{D}_{b} & \mathbf{0}_{2 \times 2} \\ \mathbf{0} & \boldsymbol{D}_{s}\end{array}\right]$,

with

$$
\boldsymbol{D}_{b}=\left[\begin{array}{ccc}
C_{11} & C_{12} & 0 \\
& C_{22} & 0 \\
\text { sym } & & C_{44}
\end{array}\right] \boldsymbol{D}_{s}=\left[\begin{array}{cc}
C_{55} & 0 \\
0 & C_{44}
\end{array}\right],
$$

$$
\begin{aligned}
C_{11}=C_{12} & =\frac{E(z)}{(1-v(z))(1+v(z))} ; \\
C_{12} & =\frac{v(z) E(z)}{(1-v(z))(1+v(z))} ; \\
C_{66} & =C_{55}=C_{44}=\frac{E(z)}{2(1+v(z))} .
\end{aligned}
$$

According to the first-order shear deformation theory (FSDT), the displacement field of the nanoplates is expressed as

$$
\boldsymbol{U}=\left\{\begin{array}{l}
u_{1}(x, y, z)=u_{0}(x, y)+z \varphi_{x}(x, y) \\
u_{2}(x, y, z)=v_{0}(x, y)+z \varphi_{y}(x, y) \\
u_{3}(x, y, z)=w_{0}(x, y)
\end{array}\right.
$$

where $u_{1}, u_{2}, u_{3}$ are the displacements at any point $(x, y, z)$; $u_{0}, v_{0}, w_{0}$ are the displacements at the mid-plane; and $\varphi_{x}$, $\varphi_{y}$ are the rotation angles of the cross-section around the $y$ -axis and $x$-axis, respectively.

The deformation field of the FG nanoplate is defined as follows:

$\boldsymbol{\varepsilon}=\left\{\begin{array}{c}\varepsilon_{x x} \\ \varepsilon_{y y} \\ \varepsilon_{x y} \\ \varepsilon_{x z} \\ \varepsilon_{y z}\end{array}\right\}=\left\{\begin{array}{c}u_{1, x} \\ v_{2, y} \\ u_{1, y}+u_{2, x} \\ u_{3, x}+u_{1, z} \\ u_{3, y}+u_{2, z}\end{array}\right\}=\left\{\begin{array}{c}u_{0, x} \\ v_{0, y} \\ u_{0, y}+v_{0, x} \\ w_{0 . x}+\varphi_{x} \\ w_{0, y}+\varphi_{y}\end{array}\right\}+z\left\{\begin{array}{c}\varphi_{x, x} \\ \varphi_{y, y} \\ \varphi_{x, y}+\varphi_{y, x} \\ 0 \\ 0\end{array}\right\}$.

Equation (11) may be written as

$$
\varepsilon=\left\{\begin{array}{c}
\varepsilon_{1} \\
\varepsilon_{2}
\end{array}\right\}=\left\{\begin{array}{c}
\varepsilon_{1}^{0}+z \varepsilon_{1}^{1} \\
\varepsilon_{2}^{0}
\end{array}\right\}
$$

with

$$
\begin{gathered}
\varepsilon_{1}=\left\{\begin{array}{c}
\varepsilon_{x x} \\
\varepsilon_{y y} \\
\varepsilon_{x y}
\end{array}\right\} ; \varepsilon_{2}=\left\{\begin{array}{c}
\varepsilon_{x z} \\
\varepsilon_{y z}
\end{array}\right\} ; \varepsilon_{1}^{0}=\left\{\begin{array}{c}
u_{o, x} \\
v_{o, y} \\
u_{0, y}+v_{0, x}
\end{array}\right\} ; \\
\varepsilon_{1}^{1}=\left\{\begin{array}{c}
\varphi_{x, x} \\
\varphi_{y, y} \\
\varphi_{x, y}+\varphi_{y, x}
\end{array}\right\} ; \varepsilon_{2}^{0}=\left\{\begin{array}{c}
w_{0, x}+\varphi_{x} \\
w_{0, y}+\varphi_{y}
\end{array}\right\} .
\end{gathered}
$$

From Equations (4)-(13), the nonlocal force and moment resultants are determined by

$$
\left\{\begin{array}{l}
N_{i j} \\
M_{i j}
\end{array}\right\}=\int_{-h / 2}^{h / 2} \sigma_{i j}\left\{\begin{array}{l}
1 \\
z
\end{array}\right\} \mathrm{d} z ; i j=x x, y y, x y,
$$




$$
\left\{\begin{array}{c}
Q_{x z} \\
Q_{y z}
\end{array}\right\}=\int_{-h / 2}^{h / 2}\left\{\begin{array}{c}
\sigma_{x z} \\
\sigma_{y z}
\end{array}\right\} \mathrm{d} z .
$$

Equations (14) and (15) can be rewritten as

$$
\begin{aligned}
& \left\{\begin{array}{lll}
N_{x x} & N_{y y} & N_{x y}
\end{array}\right\}^{T}=\boldsymbol{A} \varepsilon_{1}^{0}+\boldsymbol{B} \varepsilon_{1}^{1}, \\
& \left\{\begin{array}{lll}
M_{x x} & M_{y y} & M_{x y}
\end{array}\right\}^{T}=\boldsymbol{B} \varepsilon_{1}^{0}+\boldsymbol{F} \varepsilon_{1}^{1} \text {, } \\
& \left\{Q_{x z} Q_{y z}\right\}^{T}=A^{b} \varepsilon_{2}^{0},
\end{aligned}
$$

where

$$
(\boldsymbol{A}, \boldsymbol{B}, \boldsymbol{F})=\int_{-h / 2}^{h / 2} \boldsymbol{D}_{b}\left(1, z, z^{2}\right) \mathrm{d} z ; \boldsymbol{A}^{b}=\frac{5}{6} \int_{-h / 2}^{h / 2} \boldsymbol{D}_{s} \mathrm{~d} z,
$$

which leads to

$$
\begin{gathered}
\left\{\begin{array}{l}
N_{x x} \\
N_{y y} \\
N_{x y}
\end{array}\right\}-\mu \nabla^{2}\left\{\begin{array}{c}
N_{x x} \\
N_{y y} \\
N_{x y}
\end{array}\right\}=\boldsymbol{A} \boldsymbol{\varepsilon}_{1}^{0}+\boldsymbol{B} \boldsymbol{\varepsilon}_{1}^{1}, \\
\left\{\begin{array}{l}
M_{x x} \\
M_{y y} \\
M_{x y}
\end{array}\right\}-\mu \nabla^{2}\left\{\begin{array}{l}
M_{x x} \\
M_{y y} \\
M_{x y}
\end{array}\right\}=\boldsymbol{B} \varepsilon_{1}^{0}+\boldsymbol{F} \varepsilon_{1}^{1}, \\
\left\{\begin{array}{c}
Q_{x z} \\
Q_{y z}
\end{array}\right\}-\mu \nabla^{2}\left\{\begin{array}{c}
Q_{x z} \\
Q_{y z}
\end{array}\right\}=\boldsymbol{A}^{b} \varepsilon_{2}^{0} .
\end{gathered}
$$

By using Hamilton's principle, the equilibrium equations of the FG nanoplates are written by the following formula [49]:

$$
\begin{aligned}
N_{x x, x}+N_{x y, y} & =J_{0} \ddot{u}_{0}+J_{1} \ddot{\varphi}_{x}, \\
N_{x y, x}+N_{y y, y} & =J_{0} \ddot{v}_{0}+J_{1} \ddot{\varphi}_{y}, \\
Q_{x z, x}+Q_{y z, y}-R & =J_{0} \ddot{w}_{0}, \\
M_{x x, x}+M_{x y, y}-Q_{x z} & =J_{1} \ddot{u}_{0}+J_{2} \ddot{\varphi}_{x}, \\
M_{x y, x}+M_{y y, y}-Q_{y z} & =J_{1} \ddot{v}_{0}+J_{2} \ddot{\varphi}_{y},
\end{aligned}
$$

in which $R$ is the active force which is the combination between the external force in the $z$-direction and the reaction force of the elastic foundation as follows

$$
R=q(x, y)-k_{w} w_{0} .
$$

The mass inertia moment components are determined by the following formula:

$$
\left(J_{0}, J_{1}, J_{2}\right)=\int_{-h / 2}^{h / 2}\left(1, z, z^{2}\right) \rho(z) \mathrm{d} z .
$$

Substituting Equations (18)-(20) into Equations

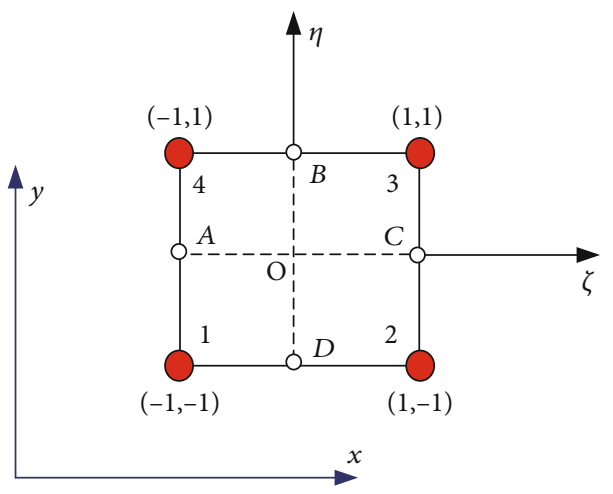

FIGURE 2: Four-node plate element.

(21)-(25), we obtain equilibrium equations of the sandwich FG nanoplates according to the nonlocal theory as follows:

$$
\begin{aligned}
N_{x x, x}+N_{x y, y} & =\left(1-\mu \nabla^{2}\right)\left(J_{0} \ddot{u}_{0}+J_{1} \ddot{\varphi}_{x}\right), \\
N_{x y, x}+N_{y y, y} & =\left(1-\mu \nabla^{2}\right)\left(J_{0} \ddot{v}_{0}+J_{1} \ddot{\varphi}_{y}\right), \\
Q_{x z, x}+Q_{y z, y} & =\left(1-\mu \nabla^{2}\right)\left(J_{0} \ddot{w}_{0}+R\right), \\
M_{x x, x}+M_{x y, y}-Q_{x z} & =\left(1-\mu \nabla^{2}\right)\left(J_{1} \ddot{u}_{0}+J_{2} \ddot{\varphi}_{x}\right), \\
M_{x y, x}+M_{y y, y}-Q_{y z} & =\left(1-\mu \nabla^{2}\right)\left(J_{1} \ddot{v}_{0}+J_{2} \ddot{\varphi}_{y}\right) .
\end{aligned}
$$

Finally, we perform a few simple calculations by multiplying the equations from Equations (28)-(32), respectively, with the variables $\delta u_{0}, \delta v_{0}, \delta w_{0}, \delta \varphi_{x}, \delta \varphi_{y}$ and integrating on the $S_{c}$ domain and adding together the sides of each equation, to give the final equation as follows:

$$
\begin{aligned}
\int_{S_{c}}( & N_{x x} \delta u_{0, x}+N_{x y}\left(\delta u_{0, y}+\delta v_{0, x}\right)+N_{y y} \delta v_{0, x}-M_{x x} \delta \varphi_{x, x} \\
& +-M_{x y}\left(\delta \varphi_{x, y}+\delta \varphi_{y, x}\right)-M_{y y} \delta \varphi_{y, y}+Q_{x z}\left(\delta \varphi_{x}+\delta w_{0, x}\right) \\
& +Q_{y z}\left(\delta \varphi_{y}+\delta w_{0, y}\right)-\left(1-\mu \nabla^{2}\right)\left(q(x, y)-k_{w} w_{0}\right) \delta w_{0} \\
& --\left(1-\mu \nabla^{2}\right) J_{2}\left(\dot{\varphi}_{x} \delta \dot{\varphi}_{x}+\dot{\varphi}_{y} \delta \dot{\varphi}_{y}\right) \\
& -\left(1-\mu \nabla^{2}\right)\left(J_{0}\left(\dot{u}_{0} \delta \dot{u}_{0}+\dot{v}_{0} \delta \dot{v}_{0}+\dot{w}_{0} \delta \dot{w}_{0}\right)\right. \\
& \left.\left.+J_{1}\left(\dot{\varphi}_{x} \delta \dot{u}_{0}+\dot{\varphi}_{y} \delta \dot{v}_{0}+\dot{u}_{0} \delta \dot{\varphi}_{x}+\dot{v}_{0} \delta \dot{\varphi}_{y}\right)\right)\right) \mathrm{d} x \mathrm{~d} y=0 .
\end{aligned}
$$

\subsection{Finite Element Formulation}

2.3.1. Basic Formulation. In this study, we use the four-node plate element, each node has 5 degrees of freedom (dof) to discretize the nanoplates. Then the nodal displacement vector can be defined as follows:

$$
\boldsymbol{d}_{e}=\left[\begin{array}{llll}
\boldsymbol{d}_{1}^{T} & \boldsymbol{d}_{2}^{T} & \boldsymbol{d}_{3}^{T} & \boldsymbol{d}_{4}^{T}
\end{array}\right]^{T},
$$


TABLE 1: Mechanical properties of the materials.

\begin{tabular}{lcccccccccc}
\hline Material \#1 & $E(\mathrm{GPa})$ & $v$ & $\rho\left(\mathrm{kg} / \mathrm{m}^{3}\right)$ & Material \#2 & $E(\mathrm{GPa})$ & $v$ & $\rho\left(\mathrm{kg} / \mathrm{m}^{3}\right)$ & $\mathrm{Material} \# 3$ & $E(\mathrm{GPa})$ & $v$ \\
\hline $\mathrm{Si}_{3} \mathrm{~N}_{4}$ & 348.43 & 0.24 & 2370 & $\mathrm{Al}_{2} \mathrm{O}_{3}$ & 380 & 0.3 & 3800 & $\mathrm{ZrO}_{2}$ & 151 & 0.3 \\
$\mathrm{SU}_{3} \mathrm{~S}_{3} \mathrm{O}_{4}$ & 201.04 & 0.30 & 8166 & $\mathrm{Al}$ & 70 & 0.3 & 2707 & $\mathrm{Al}$ & 70 & 0.3 \\
\hline
\end{tabular}

TABLE 2: The convergence of dimensionless deflection $W_{1}$ of the SSSS square plate $(a / h=10)$ under sinusoidal loads.

\begin{tabular}{|c|c|c|c|c|c|c|c|c|}
\hline \multirow{2}{*}{$\begin{array}{l}n \\
\text { Mesh }\end{array}$} & \multicolumn{2}{|c|}{0} & \multicolumn{2}{|c|}{1} & \multicolumn{2}{|c|}{4} & \multicolumn{2}{|c|}{10} \\
\hline & Present & Q4 & Present & Q4 & Present & Q4 & Present & Q4 \\
\hline $2 \times 2$ & 0.3764 & 0.1900 & 0.7461 & 0.3406 & 1.1094 & 0.5518 & 1.2702 & 0.6950 \\
\hline $4 \times 4$ & 0.3168 & 0.2284 & 0.6299 & 0.4300 & 0.9341 & 0.6683 & 1.0657 & 0.8014 \\
\hline $8 \times 8$ & 0.3006 & 0.2721 & 0.5981 & 0.5313 & 0.8865 & 0.8003 & 1.0107 & 0.9278 \\
\hline $10 \times 10$ & 0.2987 & 0.2797 & 0.5942 & 0.5497 & 0.8808 & 0.8236 & 1.0041 & 0.9493 \\
\hline $16 \times 16$ & 0.2966 & 0.2889 & 0.5901 & 0.5718 & 0.8746 & 0.8513 & 0.9970 & 0.9748 \\
\hline $18 \times 18$ & 0.2966 & 0.2902 & 0.5901 & 0.5750 & 0.8746 & 0.8553 & 0.9970 & 0.9784 \\
\hline $20 \times 20$ & 0.2966 & 0.2911 & 0.5901 & 0.5773 & 0.8746 & 0.8581 & 0.9970 & 0.9811 \\
\hline [50] & \multicolumn{2}{|c|}{0.2961} & \multicolumn{2}{|c|}{0.5890} & \multicolumn{2}{|c|}{0.8815} & \multicolumn{2}{|c|}{1.0087} \\
\hline
\end{tabular}

TABLE 3: The convergence of fundamental frequency $\Omega_{1}$ of the SSSS square plate $(a / h=10)$.

\begin{tabular}{|c|c|c|c|c|c|c|c|c|}
\hline \multirow{2}{*}{$\begin{array}{l}n \\
\text { Mesh }\end{array}$} & \multicolumn{2}{|c|}{0} & \multicolumn{2}{|c|}{1} & \multicolumn{2}{|c|}{4} & \multicolumn{2}{|c|}{10} \\
\hline & Present & Q4 & Present & Q4 & Present & Q4 & Present & Q4 \\
\hline $2 \times 2$ & 0.0764 & 0.1078 & 0.0586 & 0.0869 & 0.0506 & 0.0719 & 0.0483 & 0.0655 \\
\hline $4 \times 4$ & 0.0617 & 0.0727 & 0.0473 & 0.0572 & 0.0409 & 0.0484 & 0.0391 & 0.0451 \\
\hline $8 \times 8$ & 0.0587 & 0.0617 & 0.0449 & 0.0477 & 0.0389 & 0.0409 & 0.0372 & 0.0388 \\
\hline $10 \times 10$ & 0.0584 & 0.0603 & 0.0447 & 0.0465 & 0.0387 & 0.0400 & 0.0370 & 0.0380 \\
\hline $14 \times 14$ & 0.0580 & 0.0591 & 0.0444 & 0.0454 & 0.0384 & 0.0391 & 0.0368 & 0.0373 \\
\hline $16 \times 16$ & 0.0580 & 0.0588 & 0.0444 & 0.0451 & 0.0384 & 0.0389 & 0.0368 & 0.0372 \\
\hline $18 \times 18$ & 0.0580 & 0.0585 & 0.0444 & 0.0449 & 0.0384 & 0.0388 & 0.0368 & 0.0371 \\
\hline$[51]$ & \multicolumn{2}{|c|}{0.0577} & \multicolumn{2}{|c|}{0.0442} & \multicolumn{2}{|c|}{0.0381} & \multicolumn{2}{|c|}{0.0364} \\
\hline
\end{tabular}

TABLE 4: Dimensionless displacements and stresses of the square nanoplates ( $a / h=10$, SSSS $)$ under the sinusoidal distribution loads on the $\mathrm{EF}$ and two different nonlocal factors $\mu$.

\begin{tabular}{|c|c|c|c|c|c|c|c|}
\hline \multirow{2}{*}{ Method } & \multicolumn{4}{|c|}{$\mu=0$} & \multicolumn{3}{|c|}{$\mu=4$} \\
\hline & $K_{w}$ & $W_{3}$ & $\sigma_{x x}^{* *}(\mathrm{~h} / 2)$ & $\sigma_{x y}^{* *}(-\mathrm{h} / 3)$ & $W_{3}$ & $\sigma_{x x}^{* *}(\mathrm{~h} / 2)$ & $\sigma_{x y}^{* *}(-\mathrm{h} / 3)$ \\
\hline \multirow{2}{*}{ [52] } & 0 & 2.9603 & 19.9550 & 10.7450 & 5.2977 & 35.7108 & 19.2289 \\
\hline & 100 & 2.3290 & 15.6991 & 8.4534 & 3.5671 & 24.0455 & 12.9475 \\
\hline \multirow{2}{*}{ Q4 } & 0 & 2.7974 & 18.3797 & 9.9198 & 5.0061 & 32.8917 & 17.7522 \\
\hline & 100 & 2.2417 & 14.7287 & 7.9493 & 3.4639 & 22.7590 & 12.2834 \\
\hline \multirow{2}{*}{ Present } & 0 & 2.9760 & 19.7344 & 10.6262 & 5.3258 & 35.3161 & 19.0164 \\
\hline & 100 & 2.3500 & 15.5834 & 8.3911 & 3.6036 & 23.8962 & 12.8672 \\
\hline
\end{tabular}


TABLE 5: The variation of the dimensionless displacement of the sandwich FGM square nanoplates versus to the variations of power-law index $n$ and nonlocal factor $\mu$.

\begin{tabular}{|c|c|c|c|c|c|c|}
\hline \multirow[b]{2}{*}{$\mu$} & \multicolumn{6}{|c|}{$W_{2}$} \\
\hline & $n$ & 0 & 2.5 & 5 & 7.5 & 10 \\
\hline \multirow{5}{*}{1} & $2-1-2$ & 0.1793 & 0.2763 & 0.2889 & 0.2926 & 0.2943 \\
\hline & $2-1-1$ & 0.1792 & 0.2684 & 0.2800 & 0.2836 & 0.2852 \\
\hline & $2-2-1$ & 0.1791 & 0.2569 & 0.2687 & 0.2727 & 0.2747 \\
\hline & $1-2-1$ & 0.1791 & 0.2493 & 0.2612 & 0.2656 & 0.2679 \\
\hline & $1-8-1$ & 0.1788 & 0.2085 & 0.2139 & 0.2160 & 0.2172 \\
\hline \multirow{5}{*}{2} & $2-1-2$ & 0.2007 & 0.3031 & 0.3160 & 0.3199 & 0.3216 \\
\hline & $2-1-1$ & 0.2007 & 0.2949 & 0.3069 & 0.3106 & 0.3123 \\
\hline & $2-2-1$ & 0.2006 & 0.2829 & 0.2952 & 0.2994 & 0.3015 \\
\hline & $1-2-1$ & 0.2006 & 0.2750 & 0.2874 & 0.2920 & 0.2944 \\
\hline & $1-8-1$ & 0.2002 & 0.2321 & 0.2377 & 0.2400 & 0.2413 \\
\hline \multirow{5}{*}{3} & $2-1-2$ & 0.2206 & 0.3269 & 0.3401 & 0.3440 & 0.3457 \\
\hline & $2-1-1$ & 0.2206 & 0.3186 & 0.3308 & 0.3345 & 0.3363 \\
\hline & $2-2-1$ & 0.2205 & 0.3063 & 0.3188 & 0.3232 & 0.3253 \\
\hline & $1-2-1$ & 0.2204 & 0.2982 & 0.3109 & 0.3156 & 0.3181 \\
\hline & $1-8-1$ & 0.2201 & 0.2536 & 0.2595 & 0.2619 & 0.2632 \\
\hline \multirow{5}{*}{4} & $2-1-2$ & 0.2391 & 0.3483 & 0.3615 & 0.3655 & 0.3672 \\
\hline & $2-1-1$ & 0.2390 & 0.3398 & 0.3522 & 0.3560 & 0.3577 \\
\hline & $2-2-1$ & 0.2389 & 0.3274 & 0.3401 & 0.3445 & 0.3466 \\
\hline & $1-2-1$ & 0.2389 & 0.3191 & 0.3321 & 0.3368 & 0.3393 \\
\hline & $1-8-1$ & 0.2385 & 0.2734 & 0.2795 & 0.2819 & 0.2833 \\
\hline
\end{tabular}

where $\boldsymbol{d}_{i}$ is the displacement vector at the node of element $i,(i=\overline{1-4})$ and is expressed as

$$
\boldsymbol{d}_{i}=\left\{\begin{array}{lllll}
u_{0 i} & v_{0 i} & w_{0 i} & \varphi_{x i} & \varphi_{y i}
\end{array}\right\}
$$

The displacement field in the plate element is interpolated through the node displacement vector as

$$
\left\{\begin{array}{l}
u_{0}=\boldsymbol{N}_{u} \boldsymbol{d}_{e} ; v_{0}=\boldsymbol{N}_{v} \boldsymbol{d}_{e} ; w_{0}=\boldsymbol{N}_{w} \boldsymbol{d}_{e} \\
\varphi_{x}=\boldsymbol{N}_{\varphi x} \boldsymbol{d}_{e} ; \varphi_{y}=\boldsymbol{N}_{\varphi y} \boldsymbol{d}_{e},
\end{array}\right.
$$

where $\boldsymbol{N}_{u}, \boldsymbol{N}_{v}, \boldsymbol{N}_{w}, \boldsymbol{N}_{\varphi x}, \boldsymbol{N}_{\varphi y}$ are the shape functions in the forms of

$$
\left\{\begin{array}{l}
\boldsymbol{N}_{u}=\left[\boldsymbol{N}_{1}^{(1)} \boldsymbol{N}_{2}^{(1)} \boldsymbol{N}_{3}^{(1)} \boldsymbol{N}_{4}^{(1)}\right] ; \boldsymbol{N}_{v}=\left[\boldsymbol{N}_{1}^{(2)} \boldsymbol{N}_{2}^{(2)} \boldsymbol{N}_{3}^{(2)} \boldsymbol{N}_{4}^{(2)}\right] ; \\
\boldsymbol{N}_{w}=\left[\boldsymbol{N}_{1}^{(3)} \boldsymbol{N}_{2}^{(3)} \boldsymbol{N}_{3}^{(3)} \boldsymbol{N}_{4}^{(3)}\right] ; \boldsymbol{N}_{\varphi x}=\left[\boldsymbol{N}_{1}^{(4)} \boldsymbol{N}_{2}^{(4)} \boldsymbol{N}_{3}^{(4)} \boldsymbol{N}_{4}^{(4)}\right] ; \\
\boldsymbol{N}_{\varphi y}=\left[\boldsymbol{N}_{1}^{(5)} \boldsymbol{N}_{2}^{(5)} \boldsymbol{N}_{3}^{(5)} \boldsymbol{N}_{4}^{(5)}\right] .
\end{array}\right.
$$

The matrices $N_{i}^{(j)}(j=\overline{1-5})$ in Equation (37) have the size $(1 \times 5)$
TABLE 6: The variation of the dimensionless stress $\sigma_{x x}^{* *}(\mathrm{~h} / 2)$ of the sandwich FG square nanoplate $\left(K_{w}=10, a / h=10\right.$, SSSS $)$ versus to the variations of power-law index $n$.

\begin{tabular}{ccccccc}
\hline$\mu$ & & \multicolumn{6}{c}{$\sigma_{x x}^{* * *}(\mathrm{~h} / 2)$} \\
& $n$ & 0 & 2.5 & 5 & 7.5 & 10 \\
\hline \multirow{4}{*}{1} & $2-1-2$ & 0.8323 & 1.2996 & 1.3583 & 1.3753 & 1.3826 \\
& $2-1-1$ & 0.8315 & 1.1900 & 1.2359 & 1.2507 & 1.2579 \\
& $2-2-1$ & 0.8313 & 1.1430 & 1.1885 & 1.2042 & 1.2119 \\
& $1-2-1$ & 0.8314 & 1.1722 & 1.2296 & 1.2510 & 1.2620 \\
& $1-8-1$ & 0.8300 & 0.9748 & 1.0007 & 1.0112 & 1.0169 \\
\hline \multirow{4}{*}{2} & $2-1-2$ & 0.9320 & 1.4255 & 1.4860 & 1.5035 & 1.5109 \\
& $2-1-1$ & 0.9312 & 1.3075 & 1.3546 & 1.3699 & 1.3772 \\
& $2-2-1$ & 0.9310 & 1.2590 & 1.3058 & 1.3220 & 1.3299 \\
& $1-2-1$ & 0.9311 & 1.2932 & 1.3531 & 1.3754 & 1.3867 \\
& $1-8-1$ & 0.9295 & 1.0848 & 1.1123 & 1.1235 & 1.1295 \\
\hline \multirow{4}{*}{3} & $2-1-2$ & 1.0244 & 1.5376 & 1.5991 & 1.6167 & 1.6242 \\
& $2-1-1$ & 1.0235 & 1.4124 & 1.4601 & 1.4756 & 1.4830 \\
& $2-2-1$ & 1.0232 & 1.3630 & 1.4105 & 1.4269 & 1.4349 \\
& $1-2-1$ & 1.0234 & 1.4020 & 1.4637 & 1.4865 & 1.4982 \\
& $1-8-1$ & 1.0217 & 1.1854 & 1.2142 & 1.2259 & 1.2322 \\
\hline \multirow{4}{*}{4} & $2-1-2$ & 1.1101 & 1.6380 & 1.6999 & 1.7176 & 1.7251 \\
& $2-1-1$ & 1.1091 & 1.5067 & 1.5545 & 1.5700 & 1.5775 \\
& $2-2-1$ & 1.1089 & 1.4568 & 1.5046 & 1.5209 & 1.5289 \\
& $1-2-1$ & 1.1091 & 1.5004 & 1.5632 & 1.5864 & 1.5982 \\
& $1-8-1$ & 1.1073 & 1.2777 & 1.3076 & 1.3196 & 1.3262 \\
\hline
\end{tabular}

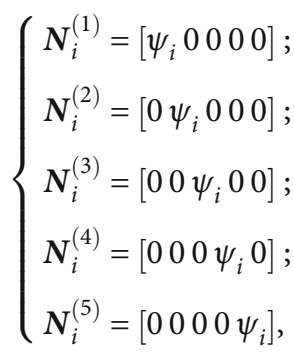

where $\psi_{i}$ are the Lagrange interpolations, and it is shown in Appendix.

Substituting Equation (36) into Equation (33), the FEM of the typical element can be expressed as

$$
M_{e} \ddot{d}_{e}+K_{e} \boldsymbol{d}_{e}=\boldsymbol{F}_{e}
$$

where $\boldsymbol{K}_{\boldsymbol{e}}$ is the element stiffness matrix defined as

$$
\boldsymbol{K}_{e}=\boldsymbol{K}_{e}^{b}+\boldsymbol{K}_{e}^{s}+\boldsymbol{K}_{e}^{f}
$$

where $\boldsymbol{K}_{e}^{b}, \boldsymbol{K}_{e}^{s}, \boldsymbol{K}_{e}^{f}$ are the bending, shear element stiffness matrices, and the foundation stiffness matrix, respectively, and are defined as

$$
\boldsymbol{K}_{e}^{b}=\int_{S_{c}}\left(\left[\boldsymbol{B}_{1}^{T} \boldsymbol{B}_{2}^{T}\right]\left[\begin{array}{ll}
\boldsymbol{A} & \boldsymbol{B} \\
\boldsymbol{B} & \boldsymbol{F}
\end{array}\right]\left[\begin{array}{l}
\boldsymbol{B}_{1} \\
\boldsymbol{B}_{2}
\end{array}\right]\right) \mathrm{d} x \mathrm{~d} y
$$



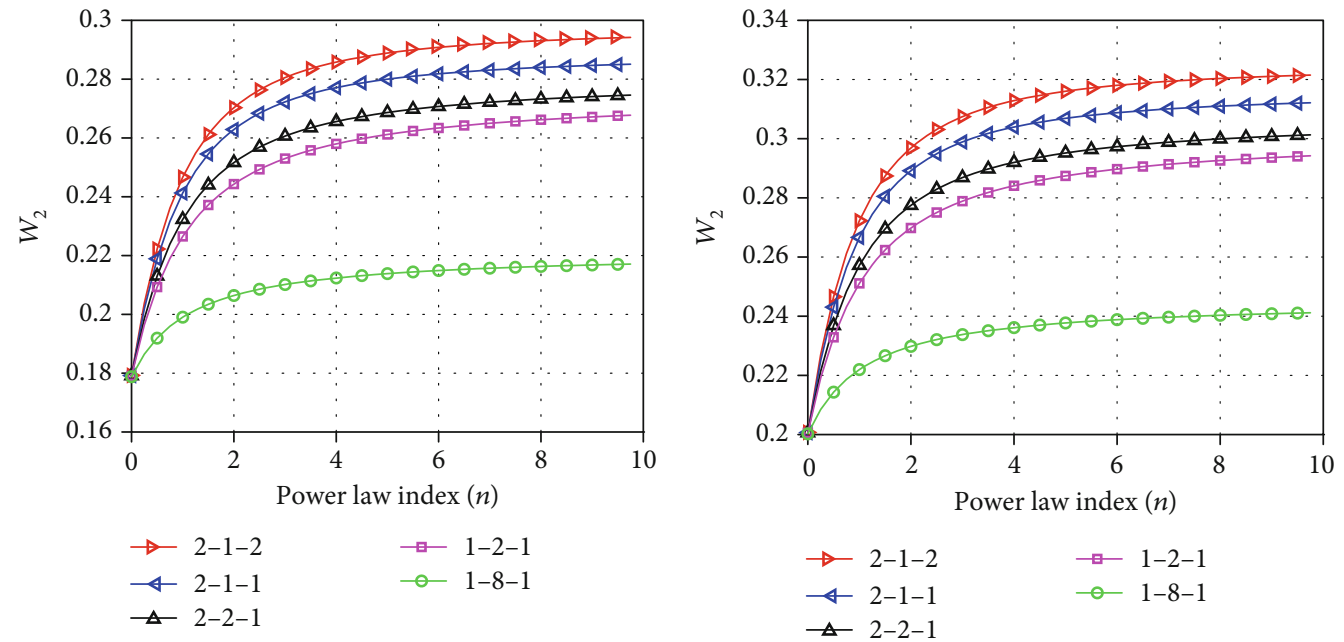

(a)
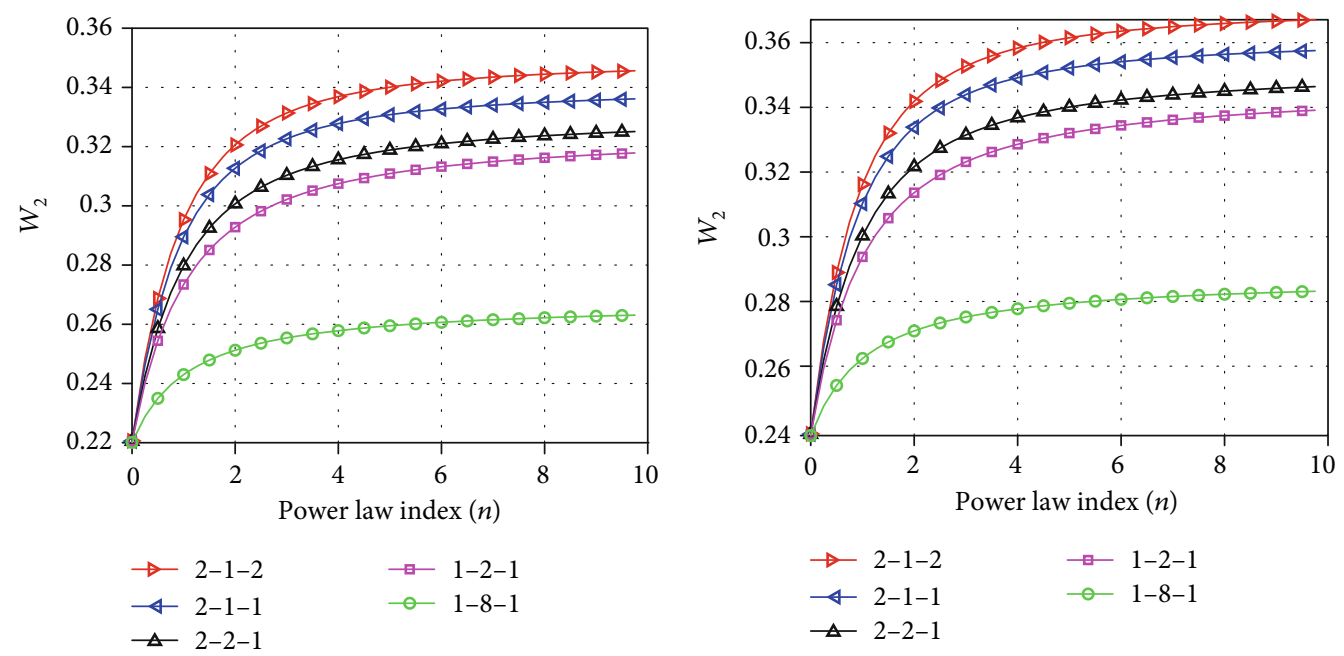

(c)

(d)

Figure 3: The variation of the dimensionless stress $\sigma_{x x}^{* *}(h / 2)$ of the sandwich FG square nanoplate versus to the variation of power-law index $n$ corresponding to different nonlocal factors $\mu$ : (a) $\mu=1$, (b) $\mu=2$, (c) $\mu=3$, (d) $\mu=4$.

$$
\begin{aligned}
\boldsymbol{K}_{e}^{s}= & \int_{S_{c}}\left(\left(\boldsymbol{B}_{3}\right)^{T} \boldsymbol{A}^{b} \boldsymbol{B}_{3}\right) \mathrm{d} x \mathrm{~d} y \\
\boldsymbol{K}_{e}^{f}= & \int_{S_{c}} k_{w}\left(\boldsymbol{B}_{w}^{T} \boldsymbol{B}_{w}+\mu\left(\boldsymbol{B}_{w x}^{T} \boldsymbol{B}_{w x}+\boldsymbol{B}_{w y}^{T} \boldsymbol{B}_{w y}\right)\right) \mathrm{d} x \mathrm{~d} y \\
\boldsymbol{M}_{e}= & \int_{S_{c}}\left(\left(\boldsymbol{N}^{T} \boldsymbol{D}_{m} \boldsymbol{N}+\mu\left(d \boldsymbol{N}_{x}^{T} \boldsymbol{D}_{m} d \boldsymbol{N}_{x}+d \boldsymbol{N}_{y}^{T} \boldsymbol{D}_{m} d \boldsymbol{N}_{y}\right)\right) \mathrm{d} x \mathrm{~d} y\right. \\
\boldsymbol{F}_{e}= & \int_{S_{c}}\left(\left(1-\mu \nabla^{2}\right) q(x, y) \boldsymbol{B}_{w}^{T}\right) d x d y \\
= & -\mu \int_{S_{c}}\left(\boldsymbol{B}_{w}^{T}(q, x x\right. \\
& \left.\left.+-\mu \int_{S_{c}}(q, y)+q_{, y y}(x, y)\right)\right) d x d y \\
& +\int_{S_{c}} q(x, y)\left(\boldsymbol{B}_{w}^{T} \mathrm{~d} x \mathrm{~d} y,\right.
\end{aligned}
$$

where

$$
\begin{aligned}
& \boldsymbol{B}_{1}=\left[\begin{array}{c}
N_{u, x} \\
N_{v, y} \\
N_{u, x}+N_{v, y}
\end{array}\right] ; \boldsymbol{B}_{2}=\left[\begin{array}{c}
N_{\varphi x, x} \\
N_{\varphi y, y} \\
N_{\varphi x, y}+N_{\varphi y, x}
\end{array}\right] ; \boldsymbol{B}_{3}=\left[\begin{array}{c}
N_{w, x}+N_{\varphi x} \\
N_{w, y}+N_{\varphi y}
\end{array}\right] ; \\
& \boldsymbol{B}_{w}=\left[\boldsymbol{N}_{w}\right] ; \boldsymbol{B}_{w x}=\left[\boldsymbol{N}_{w, x}\right] ; \boldsymbol{B}_{w y}=\left[\boldsymbol{N}_{w, y}\right] ; \boldsymbol{B}_{w, x x}=\left[\boldsymbol{N}_{w, x x}\right] ; \boldsymbol{B}_{w, y y}=\left[\boldsymbol{N}_{w, y y}\right] ; \\
& \boldsymbol{N}=\left[\boldsymbol{N}_{u}^{T} \boldsymbol{N}_{v}^{T} \boldsymbol{N}_{w}^{T} \boldsymbol{N}_{\varphi x}^{T} \boldsymbol{N}_{\varphi y}^{T}\right] ; d \boldsymbol{N}_{x}=\boldsymbol{N}_{, x} ; \mathrm{d} \boldsymbol{N}_{y}=\boldsymbol{N}_{, y}, \\
& \boldsymbol{D}_{m}=\left[\begin{array}{ccccc}
J_{0} & 0 & 0 & 0 & J_{1} \\
& J_{0} & 0 & 0 & 0 \\
& & J_{0} & 0 & 0 \\
& & & J_{2} & 0 \\
\text { sym } & & & & J_{2}
\end{array}\right] .
\end{aligned}
$$




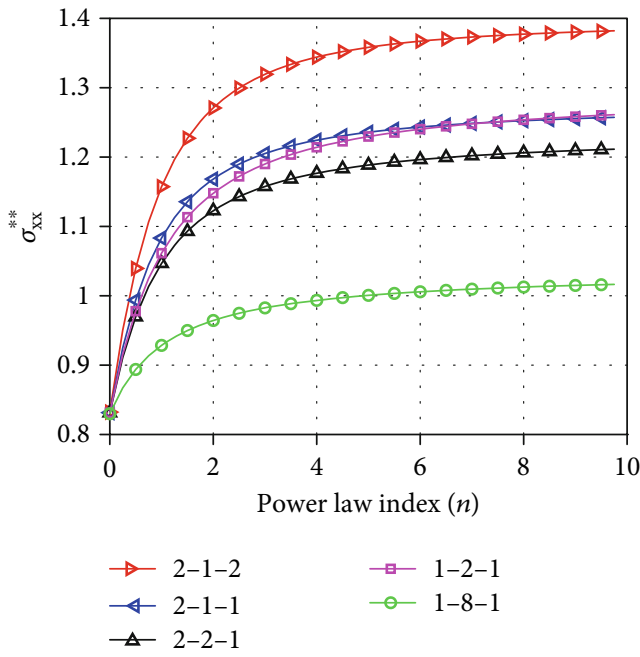

(a)

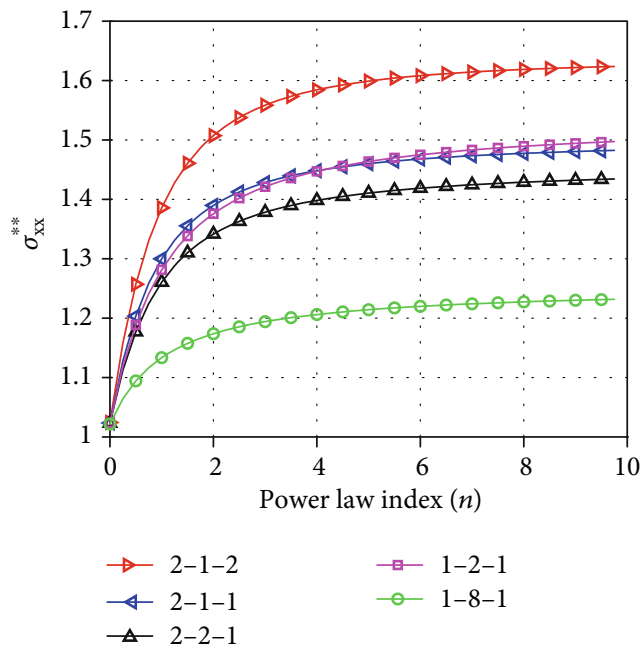

(c)

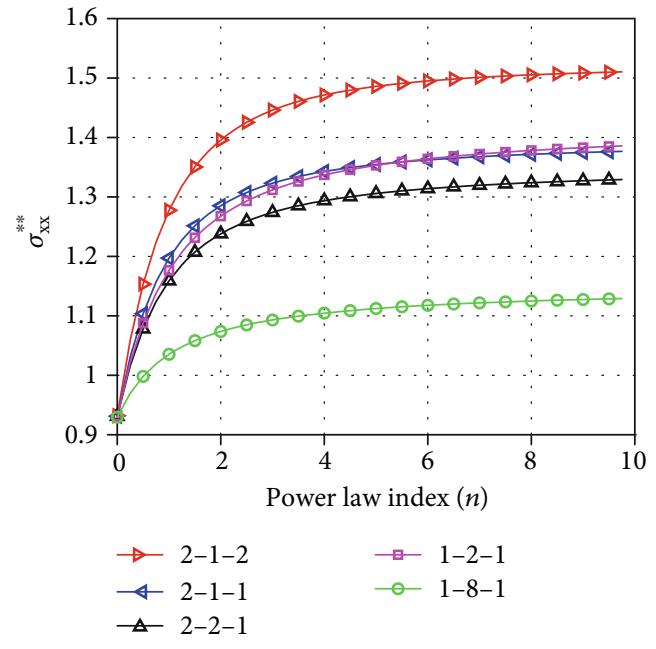

(b)

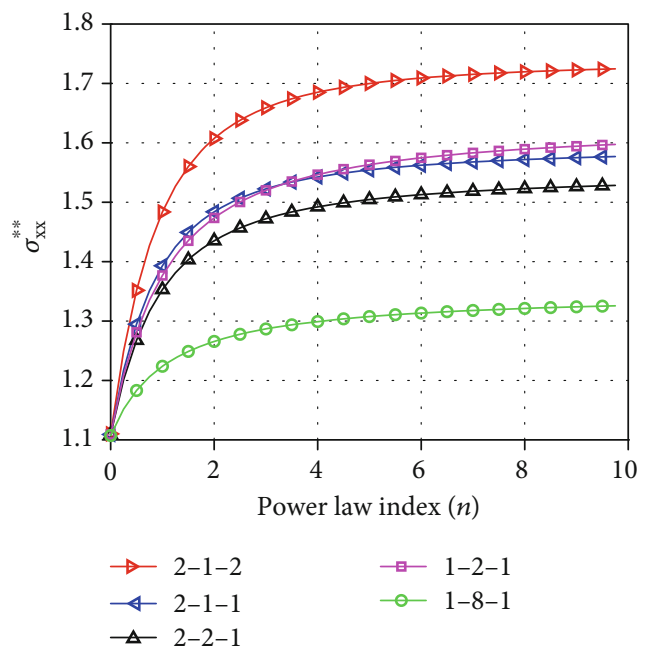

(d)

FIGURE 4: The variation of the dimensionless displacement of sandwich FG square nanoplate versus the variation of the power-law index $n$ corresponding to different nonlocal factors $\mu$ : (a) $\mu=1$, (b) $\mu=2$, (c) $\mu=3$, (d) $\mu=4$.

2.3.2. Transverse Shear Strains of the Element Based on MITC. The shear strains are approximated by interpolation in the natural coordinate system by [45]:

$$
\left\{\begin{array}{c}
\varepsilon_{x z} \\
\varepsilon_{y z}
\end{array}\right\}=\boldsymbol{H}^{-1}\left\{\begin{array}{c}
\varepsilon_{\zeta} \\
\varepsilon_{\eta}
\end{array}\right\}=\boldsymbol{H}^{-1} \tilde{\boldsymbol{L}}\left\{\begin{array}{c}
\varepsilon_{\eta}^{A} \\
\varepsilon_{\zeta}^{B} \\
\varepsilon_{\eta}^{C} \\
\varepsilon_{\zeta}^{D}
\end{array}\right\}
$$

where $\boldsymbol{H}$ is the Jacobian matrix and $A, B, C, D$ are the midpoints of the edges as shown in Figure 2; $\varepsilon_{\eta}^{A}, \varepsilon_{\zeta}^{B}, \varepsilon_{\eta}^{C}$, $\varepsilon_{\zeta}^{D}$ are the shear deformation of the points $A, B, C, D$, respectively.

$$
\begin{aligned}
& \boldsymbol{H}^{-1}=\left[\begin{array}{ll}
x_{, \zeta} & y_{, \zeta} \\
x_{, \eta} & y_{, \eta}
\end{array}\right] ; \tilde{\boldsymbol{L}}=\frac{1}{2}\left[\begin{array}{cccc}
(1-\zeta) & 0 & (1+\zeta) & 0 \\
0 & (1-\eta) & 0 & (1+\eta)
\end{array}\right], \\
& \varepsilon_{\eta}^{A}=\frac{1}{2}\left(w_{1}-w_{4}\right)+\frac{1}{2}\left(x_{1}-x_{4}\right)\left(\varphi_{x 1}+\varphi_{x 4}\right)+\frac{1}{2}\left(y_{1}-y_{4}\right)\left(\varphi_{y 1}+\varphi_{y 4}\right) ; \\
& \varepsilon_{\zeta}^{B}=\frac{1}{2}\left(w_{4}-w_{3}\right)+\frac{1}{2}\left(x_{4}-x_{3}\right)\left(\varphi_{x 4}+\varphi_{x 3}\right)+\frac{1}{2}\left(y_{4}-y_{3}\right)\left(\varphi_{y 4}+\varphi_{y 3}\right) ; \\
& \varepsilon_{\eta}^{C}=\frac{1}{2}\left(w_{3}-w_{2}\right)+\frac{1}{2}\left(x_{3}-x_{2}\right)\left(\varphi_{x 3}+\varphi_{x 2}\right)+\frac{1}{2}\left(y_{3}-y_{2}\right)\left(\varphi_{y 3}+\varphi_{y 2}\right) ; \\
& \varepsilon_{\zeta}^{D}=\frac{1}{2}\left(w_{2}-w_{1}\right)+\frac{1}{2}\left(x_{2}-x_{1}\right)\left(\varphi_{x 2}+\varphi_{x 1}\right)+\frac{1}{2}\left(y_{2}-y_{1}\right)\left(\varphi_{y 2}+\varphi_{y 1}\right) .
\end{aligned}
$$

By transforming the shear deformation according to the dof of the element displacement vector $\boldsymbol{d}_{e}$, we obtain 

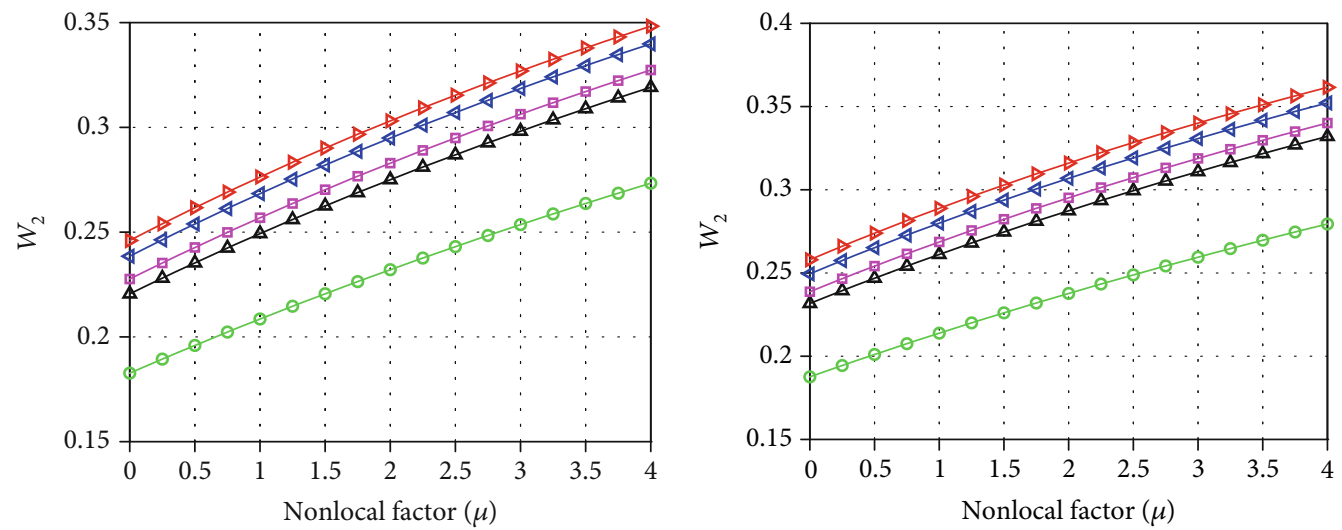

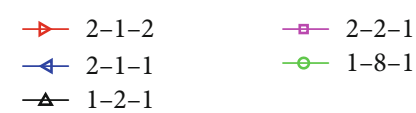

(a)

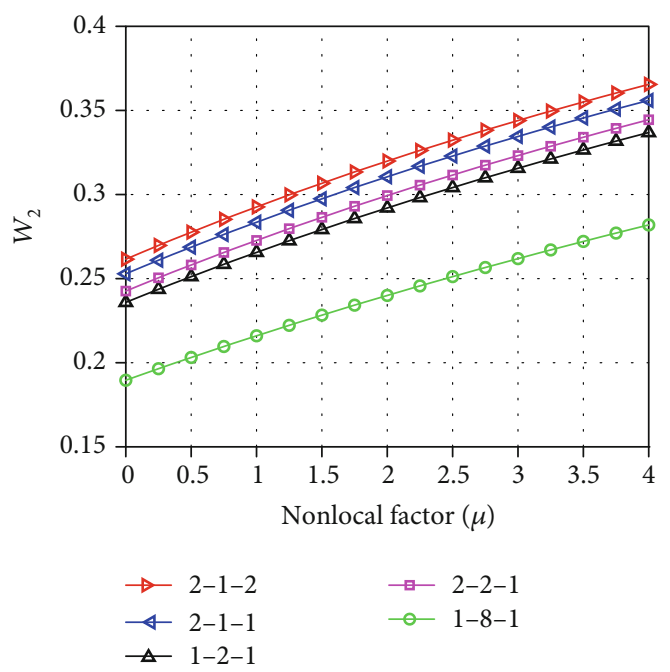

(c)

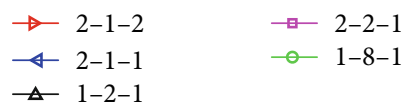

(b)

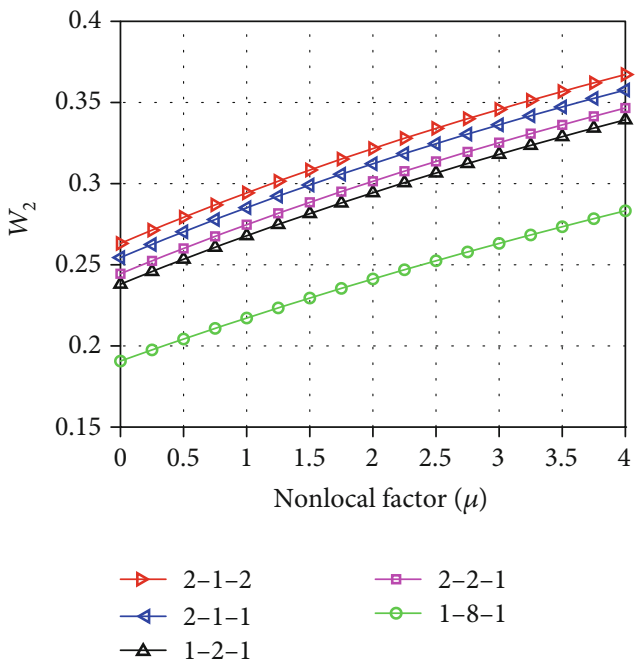

(d)

Figure 5: The variation of the dimensionless stress $\sigma_{x x}^{* *}(\mathrm{~h} / 2)$ of the sandwich FG square nanoplate versus the variation of nonlocal factor $\mu$ corresponding to different power-law indexes $n$ : (a) $n=2.5$, (b) $n=5$, (c) $n=7.5$, (d) $n=10$.

$$
\left\{\begin{array}{c}
\varepsilon_{x z} \\
\varepsilon_{y z}
\end{array}\right\}=\tilde{\boldsymbol{B}}_{3} \boldsymbol{d}_{e}
$$

where

$$
\begin{gathered}
\tilde{\boldsymbol{B}}_{3}=\boldsymbol{H}^{-1}\left[\begin{array}{llll}
\tilde{\boldsymbol{B}}_{3}^{1} & \tilde{\boldsymbol{B}}_{3}^{2} & \tilde{\boldsymbol{B}}_{3}^{3} & \tilde{\boldsymbol{B}}_{3}^{4}
\end{array}\right], \\
\tilde{\boldsymbol{B}}_{3}^{i}=\left[\begin{array}{lllll}
0 & 0 & \psi_{i, \zeta} & b_{i}^{11} \psi_{i, \zeta} & b_{i}^{12} \psi_{i, \zeta} \\
0 & 0 & \psi_{i, \eta} & b_{i}^{21} \psi_{i, \eta} & b_{i}^{22} \psi_{i, \eta}
\end{array}\right],
\end{gathered}
$$

with

$$
b_{i}^{11}=\zeta_{i} x_{, \zeta}^{M} ; b_{i}^{12}=\zeta_{i} y_{, \zeta}^{M} ; b_{i}^{21}=\eta_{i} x_{, \eta}^{L} ; b_{i}^{21}=\eta_{i} y_{, \eta}^{L}
$$

in which

$$
\begin{aligned}
\zeta_{\mathrm{i}} \in\left\{\begin{array}{llll}
-1 & 1 & 1 & -1\}
\end{array} ; \eta_{\mathrm{i}} \in\left\{\begin{array}{llll}
-1 & -1 & 1 & 1
\end{array}\right\}\right. \\
\\
(i, M, L) \in\{(1, B, A) ;(2, B, C) ;(3, D, C) ;(4, D, A)\}
\end{aligned}
$$

$$
\begin{aligned}
& \boldsymbol{r}=\left[\begin{array}{l}
x \\
y
\end{array}\right] ; \boldsymbol{r}_{, \eta}^{A}=\frac{1}{2}\left(r_{4}-r_{1}\right) ; \boldsymbol{r}_{, \eta}^{C}=\frac{1}{2}\left(r_{3}-r_{2}\right) ; \\
& \boldsymbol{r}_{, \zeta}^{B}=\frac{1}{2}\left(r_{2}-r_{1}\right) ; \boldsymbol{r}_{, \zeta}^{D}=\frac{1}{2}\left(r_{3}-r_{4}\right) .
\end{aligned}
$$

From here, the element stiffness matrix of the nanoplate can be recalculated as follows: 


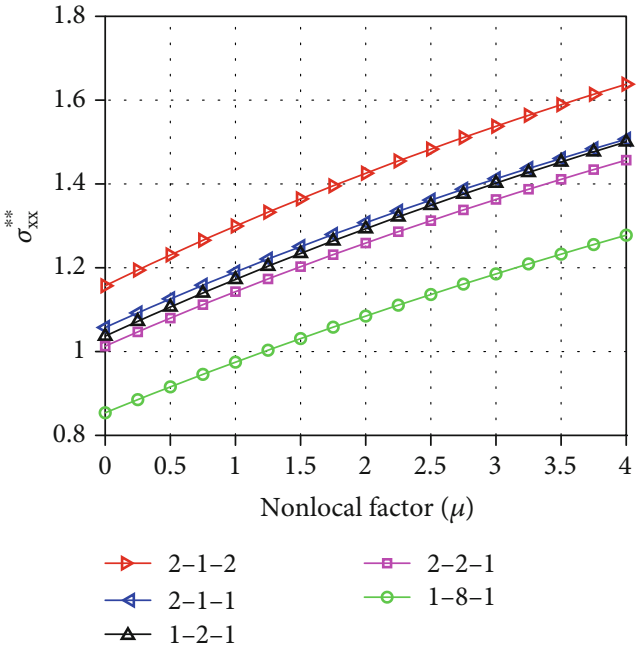

(a)

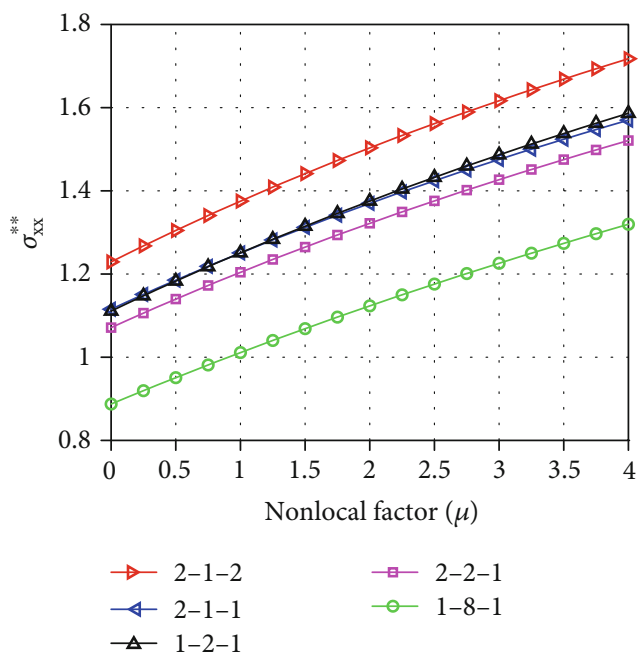

(c)

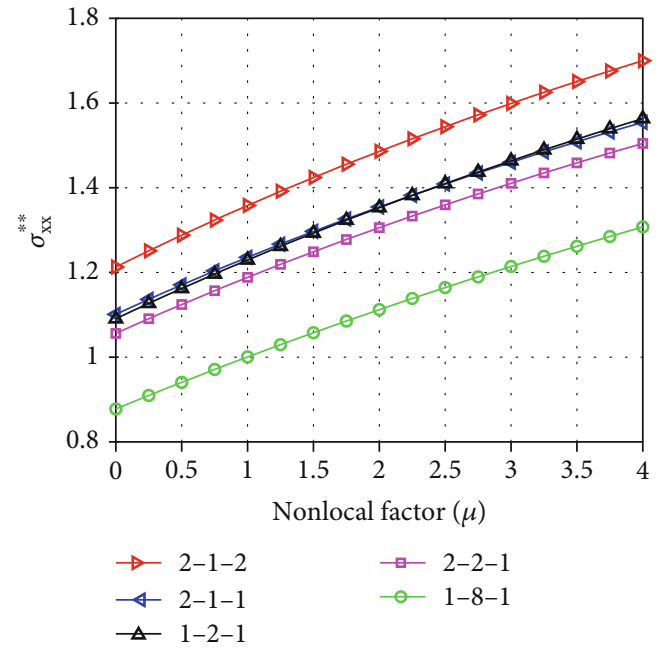

(b)

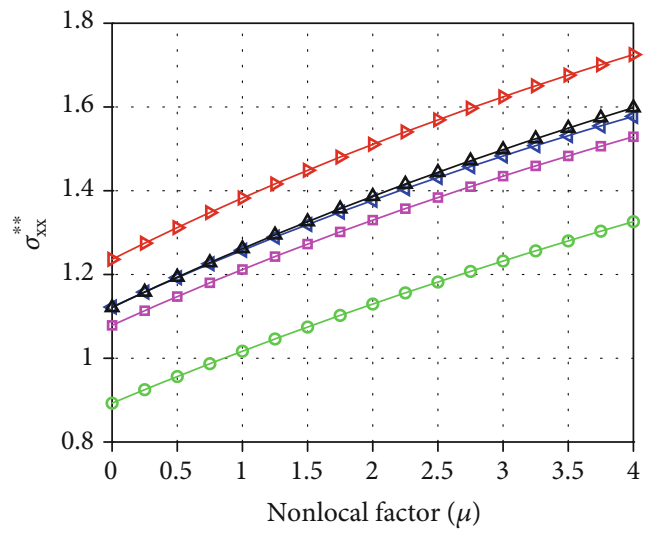

$$
\begin{array}{lll}
\rightarrow 2-1-2 & \rightarrow-2-2-1 \\
\leftarrow-2-1-1 & \rightarrow-1-8-1
\end{array}
$$

(d)

FIGURE 6: The variation of the dimensionless displacement of sandwich FG square nanoplate versus the variation of nonlocal factor $\mu$ corresponding to different power-law indexes $n$ : (a) $n=2.5$; (b) $n=5$; (c) $n=7.5$; (d) $n=10$.

$$
\begin{aligned}
\tilde{\boldsymbol{K}}_{e} & =\boldsymbol{K}_{e}^{b}+\tilde{\boldsymbol{K}}_{e}^{s}+\boldsymbol{K}_{e}^{f}, \\
\boldsymbol{K}_{e}^{b} & =\int_{S_{c}}\left(\left[\begin{array}{ll}
\boldsymbol{B}_{1}^{T} & \boldsymbol{B}_{2}^{T}
\end{array}\right]\left[\begin{array}{ll}
\boldsymbol{A} & \boldsymbol{B} \\
\boldsymbol{B} & \boldsymbol{F}
\end{array}\right]\left[\begin{array}{l}
\boldsymbol{B}_{1} \\
\boldsymbol{B}_{2}
\end{array}\right]\right) \mathrm{d} x \mathrm{~d} y, \\
\tilde{\boldsymbol{K}}_{e}^{s} & =\int_{S_{c}}\left(\left(\tilde{\boldsymbol{B}}_{3}\right)^{T} \boldsymbol{A}^{b} \tilde{\boldsymbol{B}}_{3}\right) \mathrm{d} x \mathrm{~d} y, \\
\boldsymbol{K}_{e}^{f} & =\int_{S_{c}}\left(k_{w}\left(\boldsymbol{B}_{w}^{T} \boldsymbol{B}_{w}+\mu\left(\boldsymbol{B}_{w x}^{T} \boldsymbol{B}_{w x}+\boldsymbol{B}_{w y}^{T} \boldsymbol{B}_{w y}\right)\right)\right) \mathrm{d} x \mathrm{~d} y .
\end{aligned}
$$

\section{Numerical Results}

In this section, three numerical examples are performed to demonstrate the reliability and accuracy of the proposed method. In numerical analysis, the material properties are given in Table 1. For convenience, the following dimension- less displacement, stress, and frequencies of the plate are defined as

$$
\begin{aligned}
& W_{1}=\frac{100 E_{c} h^{3}}{12\left(1-v^{2}\right) q_{0} a^{4}} w\left(\frac{a}{2}, \frac{b}{2}\right) ; W_{2}=\frac{10 E_{c} h}{q_{0} a^{2}} w\left(\frac{a}{2}, \frac{b}{2}\right) ; \\
& W_{3}=\frac{10 E_{c} h^{3}}{1 q_{0} a^{4}} w\left(\frac{a}{2}, \frac{b}{2}\right) ;
\end{aligned}
$$$$
\begin{aligned}
\sigma_{x x}^{* *}(z) & =\frac{10 h^{2}}{q_{0} a^{2}} \sigma_{x x}\left(\frac{a}{2}, \frac{b}{2}, z\right) ; \sigma_{x y}^{* *}(z)=\frac{10 h^{2}}{q_{0} a^{2}} \sigma_{x y}(0,0, z) ; \\
\Omega_{1} & =10 \omega_{11} h \sqrt{\frac{\rho_{m}}{E_{m}}}
\end{aligned}
$$

$$
\begin{aligned}
& \Omega_{2}=10 \omega_{11} \frac{a^{2}}{h} \sqrt{\frac{\rho_{0}}{E_{0}}} \text { with }: K_{w}=\frac{k_{w} a^{2}}{D_{c}} ; D_{c}=\frac{E_{c} h^{3}}{12\left(1-v^{2}\right)} ; \\
& E_{0}=1(G P a) ; \rho_{0}=1\left(\frac{\mathrm{kg}}{\mathrm{m}^{3}}\right) .
\end{aligned}
$$




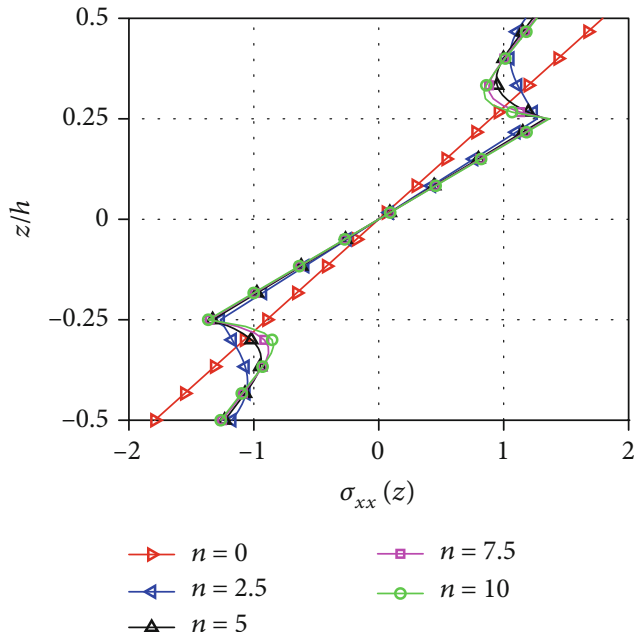

(a)

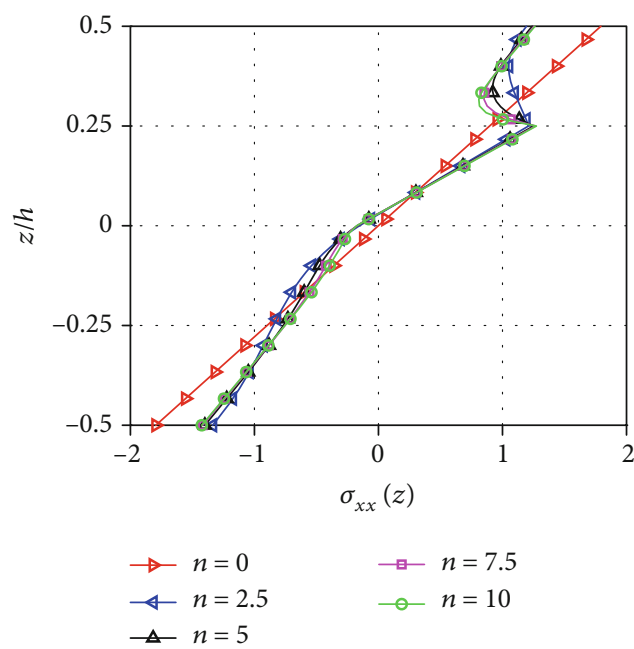

(c)

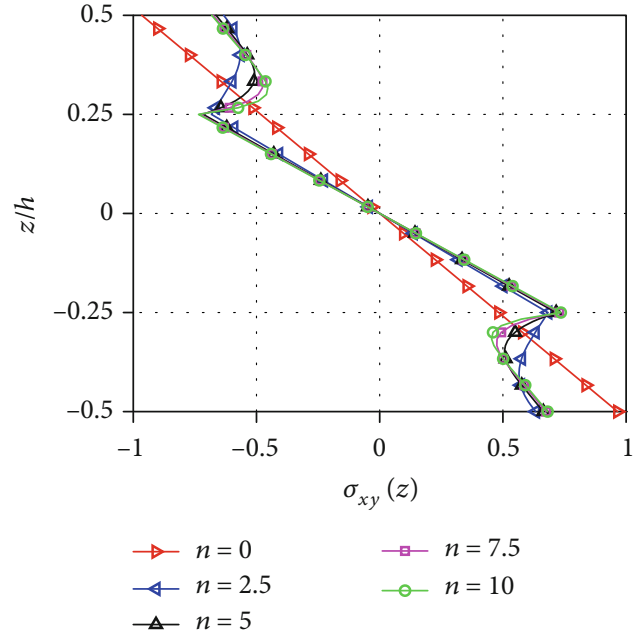

(b)

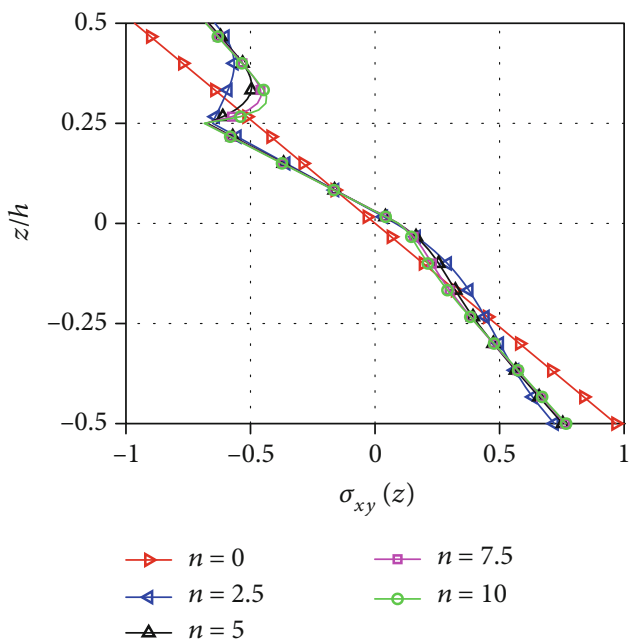

(d)

Figure 7: The variation of the dimensionless stresses of sandwich FG square nanoplate along with the thickness corresponding to two different proportions of material layers: (a) $\sigma_{x x}$ with the proportion of material layers 1-2-1; (b) $\sigma_{x y}$ with the proportion of material layers $1-2-1 ;$ (c) $\sigma_{x x}$ with the proportion of material layers 2-2-1; (d) $\sigma_{x y}$ with the proportion of material layers 2-2-1.

TABLE 7: The variation of the dimensionless displacement and the dimensionless stress $\sigma_{x x}^{* *}(h / 2)$ of the sandwich FG square nanoplate $(n$ $=2, \mu=1, a / h=10$, SSSS) versus the variation of $K_{w}$.

\begin{tabular}{|c|c|c|c|c|c|c|}
\hline & $K_{w}$ & 0 & 50 & 100 & 150 & 200 \\
\hline \multirow{5}{*}{$W_{2}$} & $2-1-2$ & 0.4261 & 0.4080 & 0.3815 & 0.3649 & 0.2864 \\
\hline & $2-1-1$ & 0.3307 & 0.3197 & 0.3033 & 0.2927 & 0.2399 \\
\hline & $2-2-1$ & 0.2703 & 0.2629 & 0.2516 & 0.2443 & 0.2064 \\
\hline & $1-2-1$ & 0.2285 & 0.2232 & 0.2150 & 0.2097 & 0.1811 \\
\hline & $1-8-1$ & 0.1979 & 0.1939 & 0.1877 & 0.1836 & 0.1614 \\
\hline \multirow{5}{*}{$\sigma_{x x}^{* *}(h / 2)$} & $2-1-2$ & 2.0033 & 1.8131 & 1.7022 & 1.7146 & 1.3380 \\
\hline & $2-1-1$ & 1.5551 & 1.4210 & 1.3530 & 1.3751 & 1.1209 \\
\hline & $2-2-1$ & 1.2708 & 1.1683 & 1.1227 & 1.1479 & 0.9644 \\
\hline & $1-2-1$ & 1.0744 & 0.9919 & 0.9594 & 0.9851 & 0.8462 \\
\hline & $1-8-1$ & 0.9305 & 0.8618 & 0.8376 & 0.8627 & 0.7539 \\
\hline
\end{tabular}




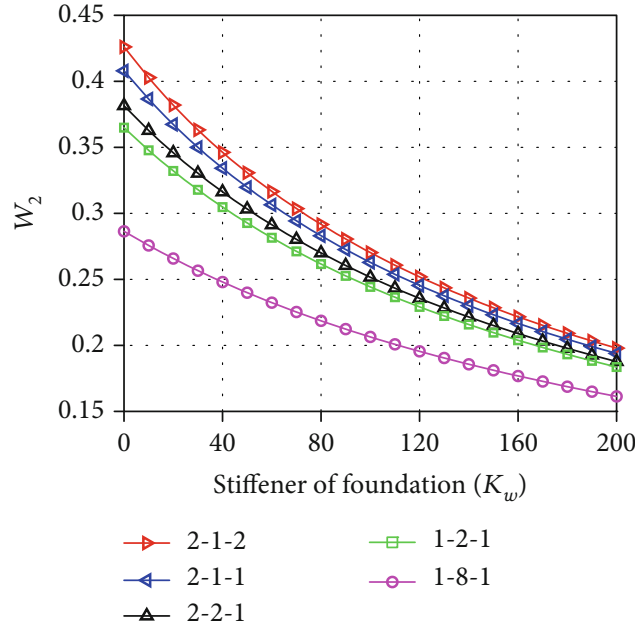

(a)

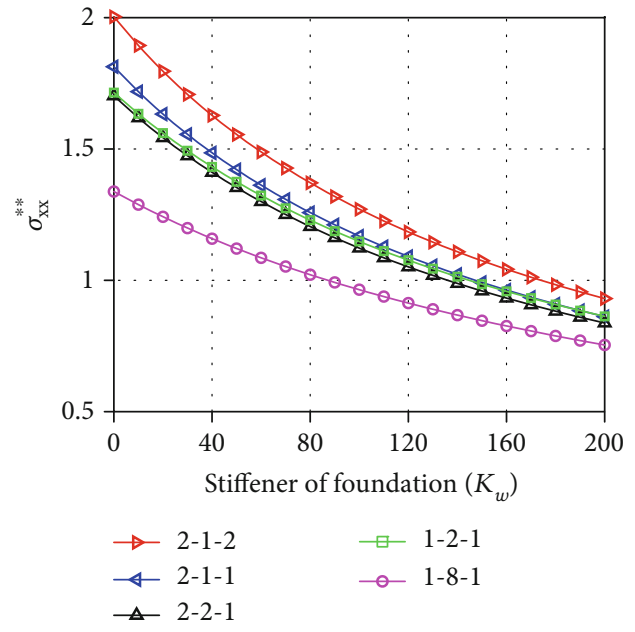

(b)

FIGURE 8: The variation of the dimensionless displacement and stress of sandwich FG square nanoplate along with thickness versus the variation of $K_{w}$ : (a) dimensionless displacement; (b) dimensionless stress.
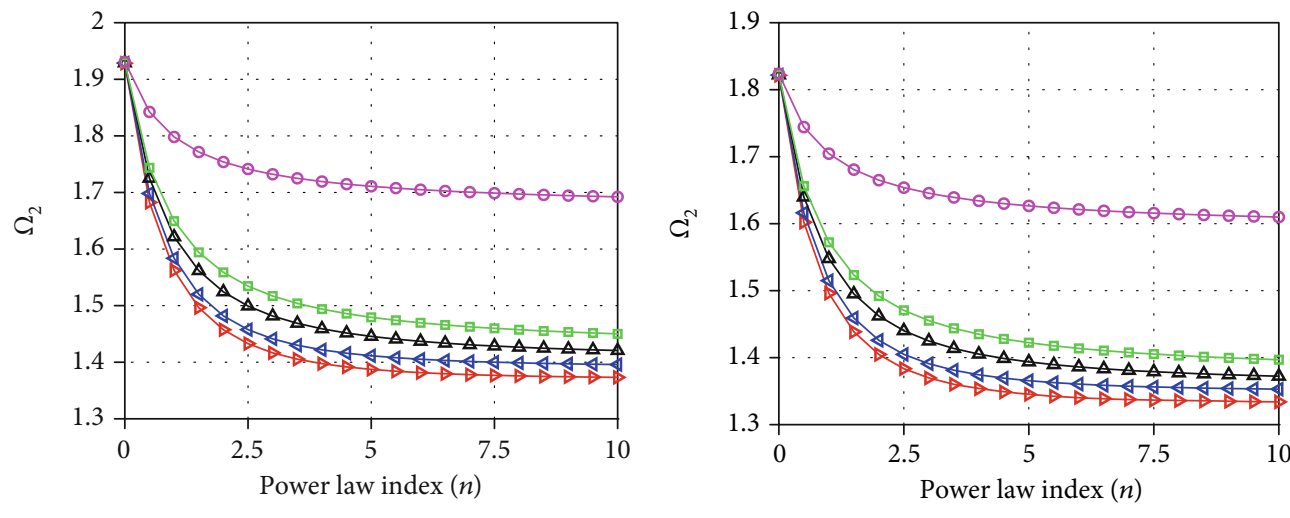

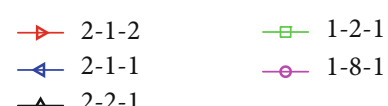

(a)

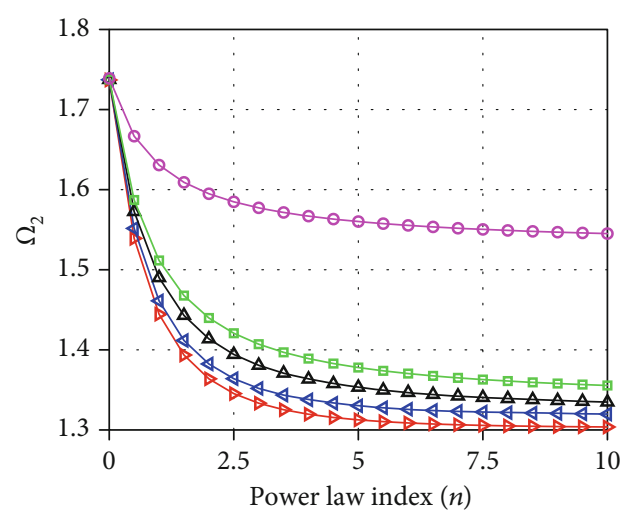

$$
\begin{array}{ll}
\rightarrow \text { 2-1-2 } & \square \text { 1-2-1 } \\
\triangle \text { 2-1-1 } & -1-8-1
\end{array}
$$$$
\triangle 2-2-
$$

(c)

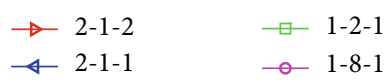

(b)

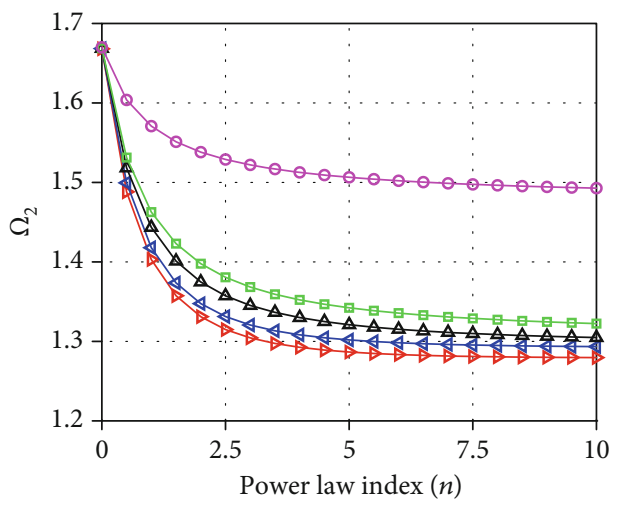

$$
\begin{aligned}
& \rightarrow \text { 2-1-2 } \quad \square \text { 1-2-1 } \\
& \text {-4 2-1-1 } \\
& \text { - } 1-8-1
\end{aligned}
$$

(d)

Figure 9: The variation of the dimensionless frequencies of the sandwich FG nanoplate versus the variation of power-law index $n$ corresponding to different nonlocal factors $\mu$; (a) $\mu=1$; (b) $\mu=2$; (c) $\mu=3$; (d) $\mu=4$. 
TABLE 8: The variation of the dimensionless frequencies of sandwich FG square nanoplate $\left(K_{w}=100, a / h=10\right.$, SSSS $)$ versus the variations of power-law index $n$.

\begin{tabular}{|c|c|c|c|c|c|c|}
\hline $\begin{array}{l}\Omega_{2} \\
\mu\end{array}$ & $n$ & 0 & 2.5 & 5 & 7.5 & 10 \\
\hline \multirow{5}{*}{1} & $2-1-2$ & 1.9283 & 1.4328 & 1.3874 & 1.3768 & 1.3732 \\
\hline & $2-1-1$ & 1.9286 & 1.4575 & 1.4114 & 1.4000 & 1.3959 \\
\hline & $2-2-1$ & 1.9290 & 1.4992 & 1.4456 & 1.4285 & 1.4207 \\
\hline & $1-2-1$ & 1.9292 & 1.5345 & 1.4794 & 1.4598 & 1.4502 \\
\hline & $1-8-1$ & 1.9307 & 1.7414 & 1.7109 & 1.6988 & 1.6922 \\
\hline \multirow{5}{*}{2} & $2-1-2$ & 1.8215 & 1.3836 & 1.3452 & 1.3366 & 1.3340 \\
\hline & $2-1-1$ & 1.8218 & 1.4049 & 1.3656 & 1.3562 & 1.3530 \\
\hline & $2-2-1$ & 1.8221 & 1.4402 & 1.3936 & 1.3790 & 1.3723 \\
\hline & $1-2-1$ & 1.8223 & 1.4706 & 1.4223 & 1.4053 & 1.3969 \\
\hline & $1-8-1$ & 1.8237 & 1.6538 & 1.6266 & 1.6157 & 1.6099 \\
\hline \multirow{5}{*}{3} & $2-1-2$ & 1.7369 & 1.3454 & 1.3126 & 1.3057 & 1.3037 \\
\hline & $2-1-1$ & 1.7372 & 1.3640 & 1.3302 & 1.3224 & 1.3198 \\
\hline & $2-2-1$ & 1.7375 & 1.3942 & 1.3533 & 1.3405 & 1.3348 \\
\hline & $1-2-1$ & 1.7376 & 1.4207 & 1.3779 & 1.3629 & 1.3555 \\
\hline & $1-8-1$ & 1.7390 & 1.5848 & 1.5602 & 1.5504 & 1.5452 \\
\hline \multirow{5}{*}{4} & $2-1-2$ & 1.6681 & 1.3149 & 1.2866 & 1.2810 & 1.2796 \\
\hline & $2-1-1$ & 1.6684 & 1.3313 & 1.3019 & 1.2954 & 1.2934 \\
\hline & $2-2-1$ & 1.6686 & 1.3574 & 1.3210 & 1.3098 & 1.3048 \\
\hline & $1-2-1$ & 1.6688 & 1.3806 & 1.3423 & 1.3289 & 1.3224 \\
\hline & $1-8-1$ & 1.6700 & 1.5289 & 1.5065 & 1.4976 & 1.4928 \\
\hline
\end{tabular}

3.1. Convergence and Accuracy Study. Firstly, the FG nanoplate using the material \#2 but with the nonlocal factor $\mu=0$ is considered. The numerical results with other mesh sizes are presented in Tables 2 and 3 and compared with the analytical method in Refs. [50, 51]. It can be seen that the results using the MITC4 element converge faster than those using the Q4 element in both static and free vibration problems. In the static bending problem, the results of proposed method using $16 \times 16$ mesh size are as accurate as those in Ref. [50], while in the free vibration problem, we only need to use $14 \times 14$ mesh size to ensure the same accuracy as in Ref. [51]. Hence, to ensure the accuracy of the proposed method, the paper will use the mesh element $16 \times 16$ to analyze both static bending and free vibration responses of nanoplates.

Next, Table 4 presents the results of the static bending analysis of the square nanoplates on the EF including the dimensionless displacement $W_{3}$, dimensionless stress $\sigma_{x x}^{* *}(h / 2)$, and $\sigma_{x y}^{* *}(-h / 3)$. It is seen that the obtained results of the present method match well with the analytical method in Ref. [52]. These results hence demonstrate the reliability and accuracy of the proposed method.

3.2. Static Bending Problem. We now investigate the static bending response of the fully simple-supported (SSSS) sandwich (Type A) FG nanoplate resting on the Winkler foundation under the sinusoidal load $q(x, y)=q_{0} \sin (\pi x / a)$ $\sin (\pi y / b)$. The dimensions of the nanoplate are given by $a=b=10 \mathrm{~nm}, h=a / 10$, and the material property is chosen by $\mathrm{Al} / \mathrm{ZrO}_{2}$ (material \#3) as shown in Table 1 . The power-law index $n$ varies from 0 to 10 , and the nonlocal factor $\mu$ varies from 1 to 4 . Five cases of the ratio of layer thickness, 2-1-2, 2-1-1, 2-2-1, 1-2-1, and 1-8-1 together with the foundation stiffness $K_{w}=100$, are considered.

Tables 5 and 6 present the numerical results of the dimensionless displacement and stresses. Figures 3 and 4 illustrate the variation of the dimensionless displacement of the nanoplate center $W_{2}$ and the dimensionless stresses when $n$ varies from 0 to 10 corresponding to $\mu=1,2,3,4$. Figures 5 and 6 present the variation of the dimensionless displacement and stresses when $\mu$ varies from 0 to 4 corresponding to $n=1,2.5,5,7.5$, and 10 . It can be seen that the increase of power-law index $n$ leads to the increase of the metal content in the FG structures and makes the FG nanoplates become softer. This result hence leads to the increase of the displacement and stress of the nanoplates. With the same power-law index $n$, the increase of the geometric parameter and of the nonlocal factor $\mu$ leads to the increase of the dimensionless displacement and stress of the FG nanoplates. Furthermore, the dimensionless displacement and stress of the sandwich FG nanoplate obtain the minimum values in the case of the ratio of layers thickness 1-8-1 and obtain the maximum values in the case of the ratio of layers thickness 2-1-2. These results show that the sandwich FG nanoplate with a constant thickness will have the higher stiffness corresponding to the increase of the thickness of the core layer (ceramic). 


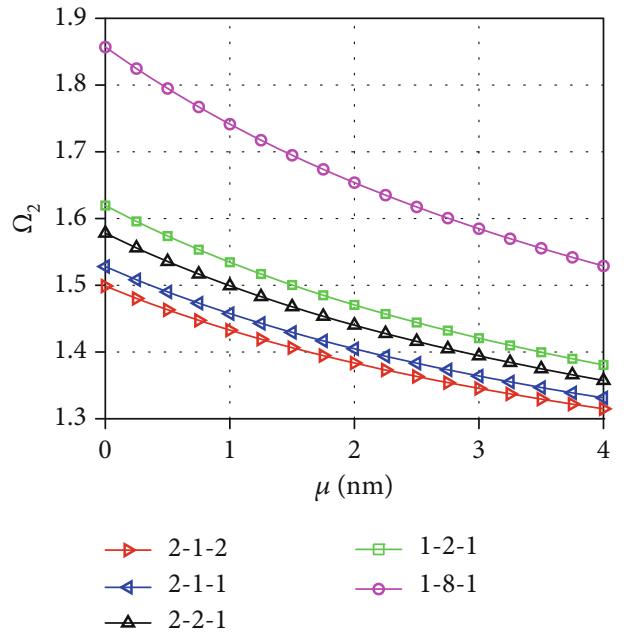

(a)

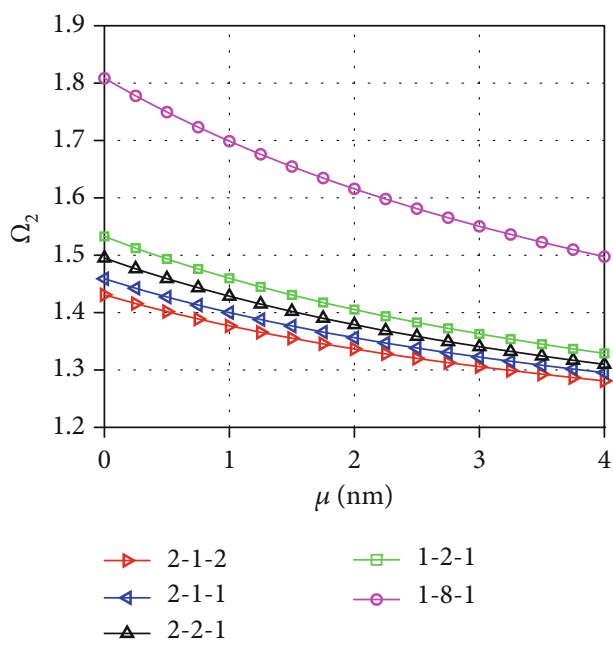

(c)

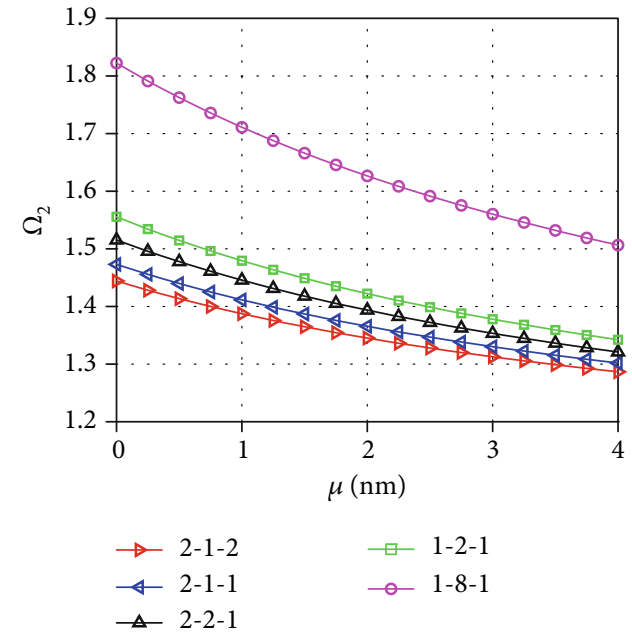

(b)

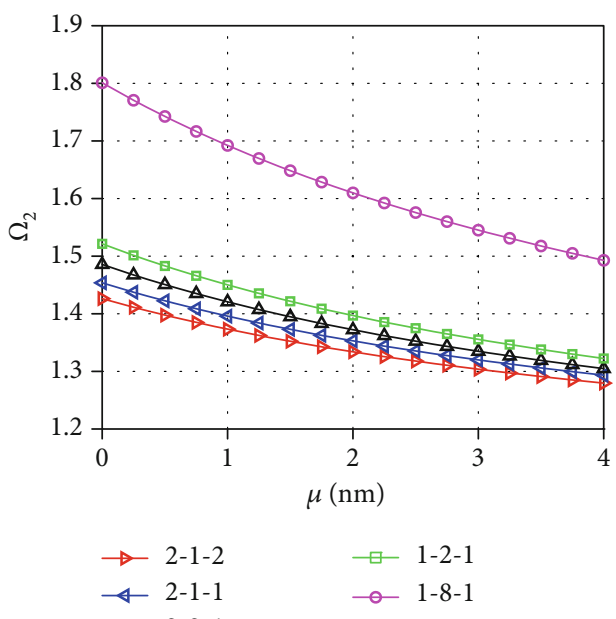

(d)

FIGURE 10: The variation of the dimensionless frequencies of the sandwich FG nanoplate versus the variation of nonlocal factor $\mu$ corresponding to different power-law indexes $n$ : (a) $n=2.5$; (b) $n=5$; (c) $n=7.5$; (d) $n=10$.

Figure 7 shows the variation of the dimensionless stress of sandwich FG square nanoplate along with the thickness corresponding to two different proportions of material layer, 1-2-1 and 2-2-1, and corresponding to the variation of power-law indexes $n=0,2.5,5,7.5,10$. Note that the nonlocal factor $\mu=1$ is fixed. It is seen that when the power-law index equals zero $(n=0)$, the sandwich FG nanoplate becomes an isotropic nanoplate, and hence the stress distributions through the thickness of FG nanoplates are linear. When the power-law indexes are different from zero $(n \neq 0)$, the stress distributions through the thickness of FG nanoplates will vary according to the folding curve types. When the core layer is homogeneous ceramic, the stress will have the jumping step at the contact surface of the middle layer with the top and bottom layers.

The effects of the foundation stiffness $K_{w}$ on the static bending response of the sandwich FG nanoplates are presented in Table 7, and the graphs of dimensionless displace- ments and stresses are shown in Figure 8. It can be seen that the increase of $K_{w}$ (from 0 to 200) leads to the decrease of the dimensionless displacement and stress for all cases of the nanoplate thickness.

3.3. Free Vibration Problem. In this example, we consider the free vibration response of a sandwich FG nanoplate (Type A) with geometric dimensions $a=b=10 \mathrm{~nm}, h=a / 10$, resting on the Winkler foundation. Figure 9 shows the numerical results of the dimensionless frequencies of the FG nanoplates when the volume fraction exponent $n$ varies from 0 to 10 with $\mu=1,2,3,4$. It can be seen that the increase of the volume fraction exponents $n$ leads to the decrease of the dimensionless frequencies. Table 8 and Figure 10 present the numerical results of the dimensionless frequencies of the FG nanoplates with the nonlocal factor $\mu$ varying from 0 to 4 and the volume fraction exponent $n=1,2.5,5,7.5,10$ corresponding to five cases of the ratio of layers thickness. 


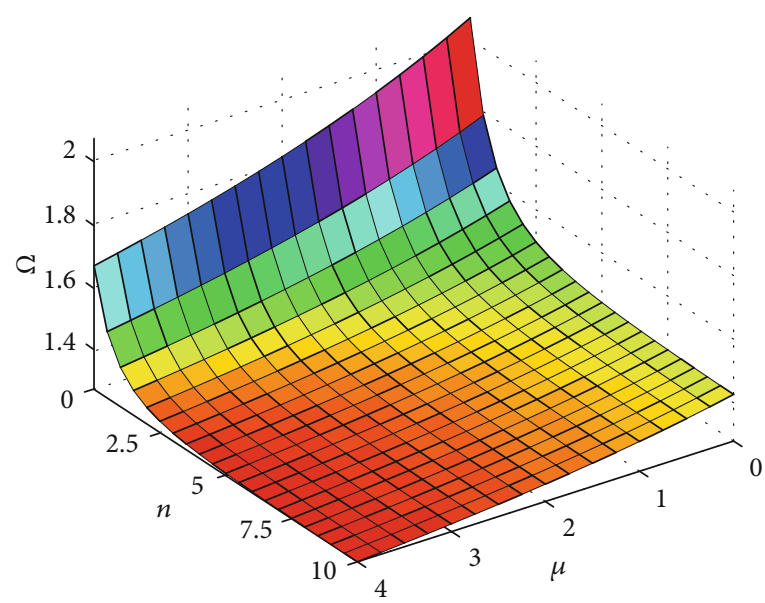

(a)

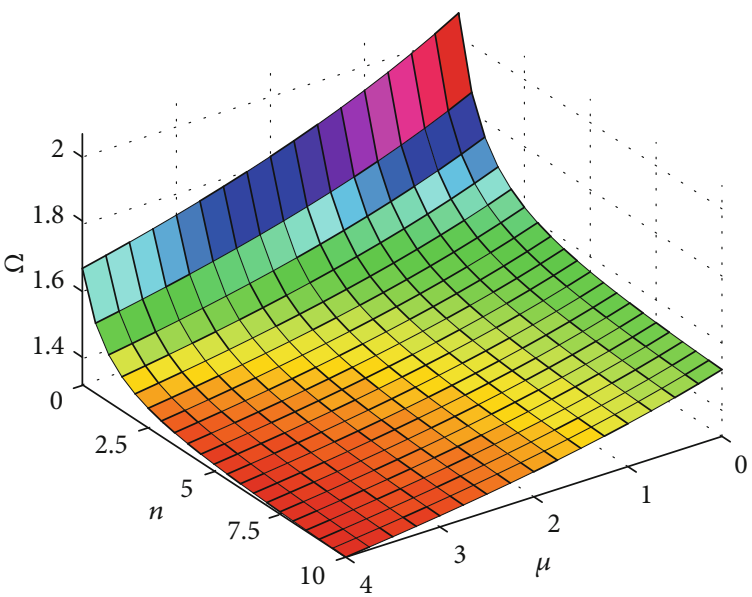

(c)

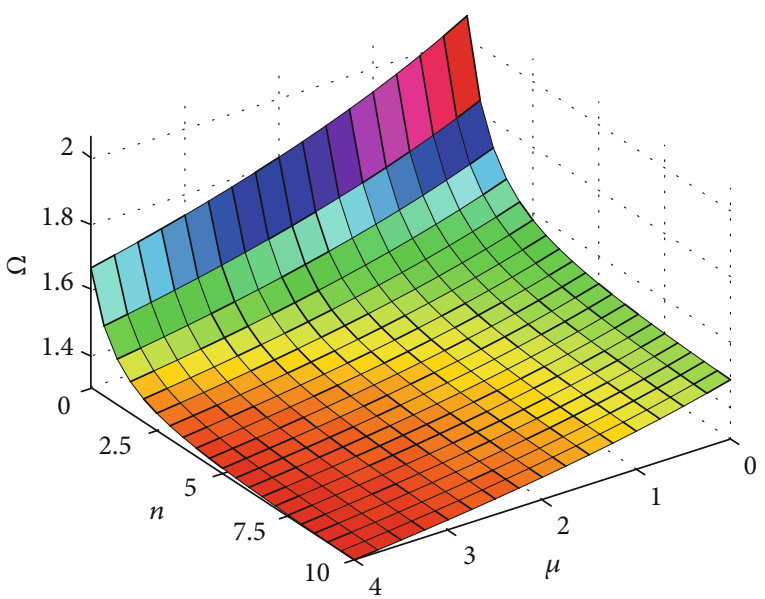

(b)

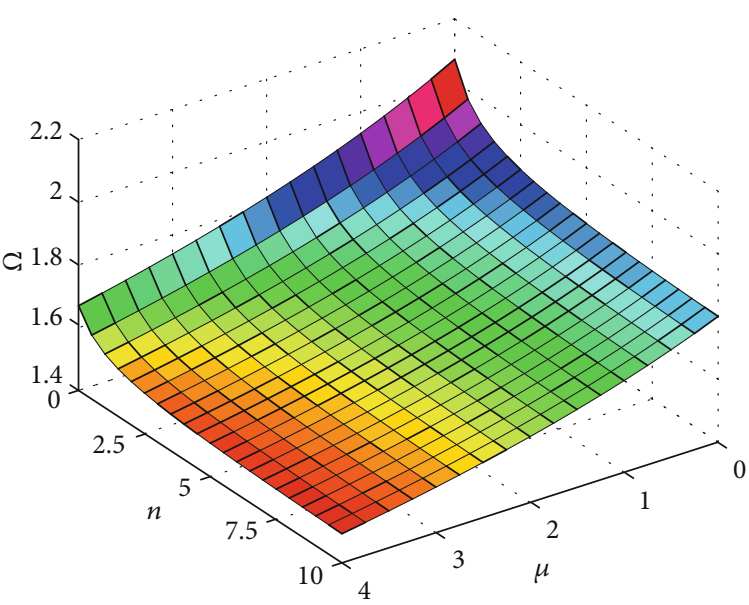

(d)

FIGURE 11: Dimensionless displacement of the sandwich FG square nanoplates with the variation of nonlocal factor $\mu$ and power-law index $n$ corresponding to different proportions of material layers: (a) 2-1-2; (b) 2-2-1; (c) 1-2-1; (d) 1-8-1.

TABLE 9: The variation of the dimensionless frequencies of sandwich FG nanoplate versus the variations of $K_{w}(n=2, \mu=1, a / h=10)$ and the different boundary conditions.

\begin{tabular}{|c|c|c|c|c|c|c|}
\hline & $K_{w}$ & 0 & 50 & 100 & 150 & 200 \\
\hline \multirow{5}{*}{ SSSS } & $2-1-2$ & 1.0299 & 1.2619 & 1.4574 & 1.6296 & 1.7853 \\
\hline & $2-1-1$ & 1.0700 & 1.2924 & 1.4819 & 1.6497 & 1.8019 \\
\hline & $2-2-1$ & 1.1430 & 1.3472 & 1.5243 & 1.6829 & 1.8278 \\
\hline & $1-2-1$ & 1.1974 & 1.3899 & 1.5588 & 1.7111 & 1.8509 \\
\hline & $1-8-1$ & 1.4629 & 1.6150 & 1.7539 & 1.8826 & 2.0031 \\
\hline \multirow{5}{*}{ SCSC } & $2-1-2$ & 1.4671 & 1.6379 & 1.7925 & 1.9348 & 2.0673 \\
\hline & $2-1-1$ & 1.5225 & 1.6859 & 1.8348 & 1.9725 & 2.1012 \\
\hline & $2-2-1$ & 1.6248 & 1.7741 & 1.9118 & 2.0401 & 2.1609 \\
\hline & $1-2-1$ & 1.7005 & 1.8408 & 1.9712 & 2.0934 & 2.2089 \\
\hline & $1-8-1$ & 2.0604 & 2.1708 & 2.2759 & 2.3763 & 2.4726 \\
\hline \multirow{5}{*}{ СССС } & $2-1-2$ & 1.7912 & 1.9332 & 2.0655 & 2.1898 & 2.3075 \\
\hline & $2-1-1$ & 1.8581 & 1.9939 & 2.1210 & 2.2408 & 2.3546 \\
\hline & $2-2-1$ & 1.9824 & 2.1062 & 2.2231 & 2.3342 & 2.4402 \\
\hline & $1-2-1$ & 2.0741 & 2.1903 & 2.3007 & 2.4060 & 2.5069 \\
\hline & $1-8-1$ & 2.5060 & 2.5974 & 2.6856 & 2.7711 & 2.8539 \\
\hline
\end{tabular}




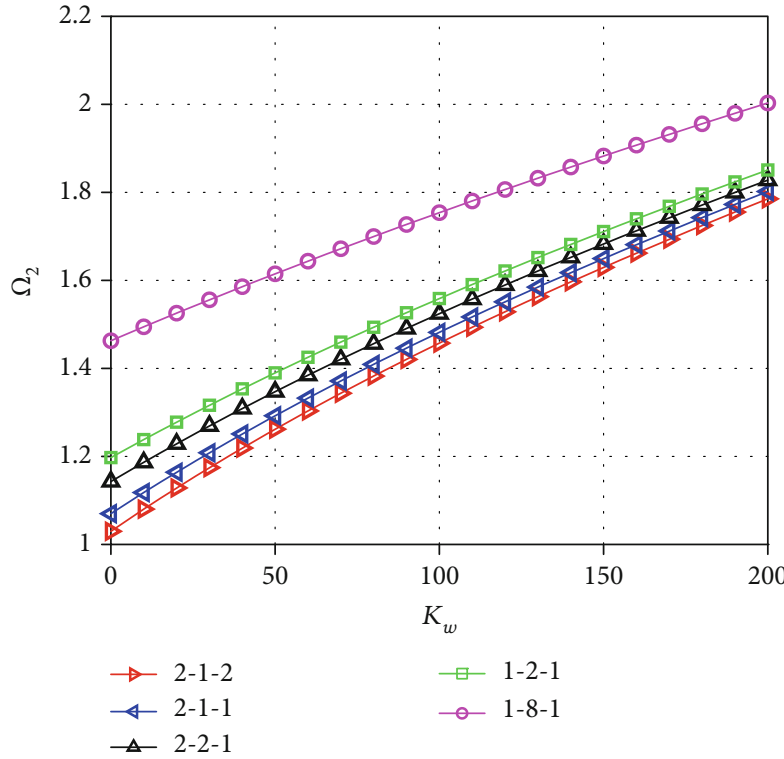

(a)

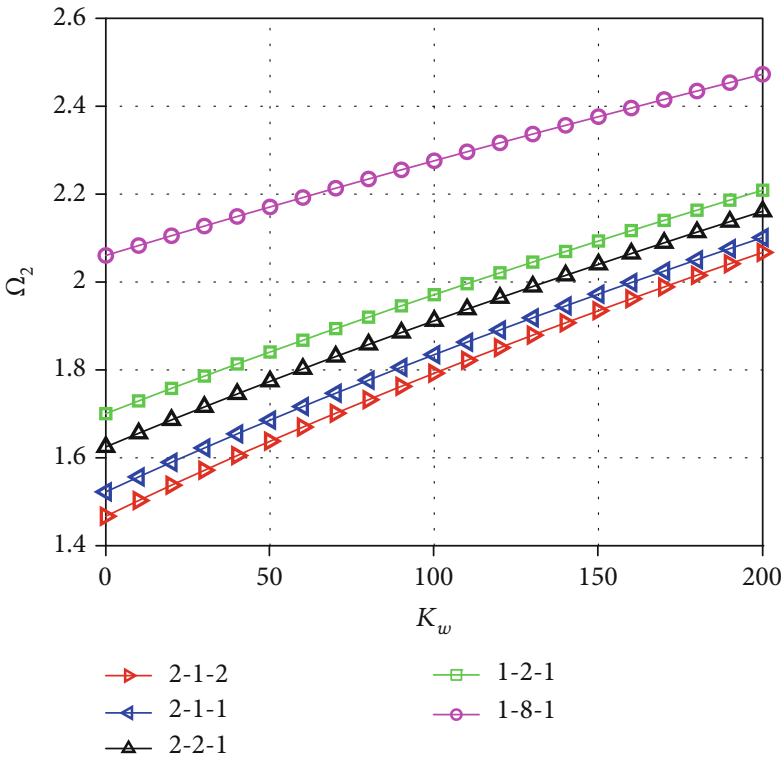

(b)

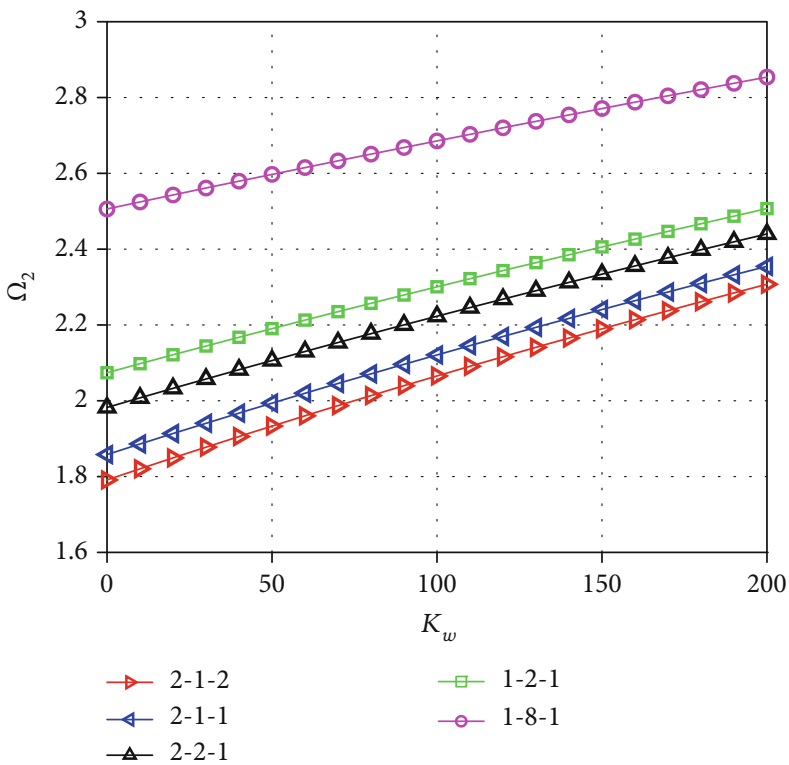

(c)

FIGURE 12: The variation of the dimensionless frequencies of the sandwich FG nanoplate through thickness versus the variation of boundary conditions: (a) SSSS; (b) SCSC; (c) CCCC.

It is noted that the increase of the nonlocal factor leads to the decrease of the dimensionless frequencies. Furthermore, Figure 11 presents the variation of the dimensionless frequencies of the FG nanoplates versus the variations of the volume fraction exponent $n$ and the nonlocal factor $\mu$. It can be observed that the increase of these values leads to the decrease of the dimensionless frequencies.

Table 9 and Figure 12 demonstrate the effects of the foundation stiffness $K_{w}$ (varying from 0 to 200) on the free vibration of the sandwich FG nanoplates. It is found that the increase of the foundation stiffness $K_{w}$ leads to the increase of the dimensionless frequencies of the FG nanoplates. In addition, the natural frequencies of the CCCC nanoplates are larger than those of the SSSS sandwich FG nanoplate. The results are quite reasonable because the SSSS boundary condition inherently offers more flexible boundary conditions than the CCCC boundary condition. Figure 13 shows the first six mode shapes of the SSSS sandwich FG square nanoplate with $K_{w}=100, n=2, \mu=1, a / h=10$. It can be seen that the second and third mode shapes are similar to each other (the second fundamental frequency is approximated the third fundamental frequency). This phenomenon is suitable for the actual symmetrical plates under the same boundary conditions. 


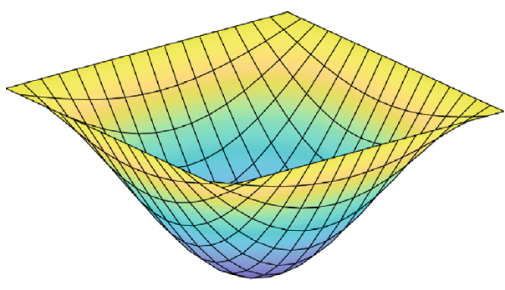

(a)

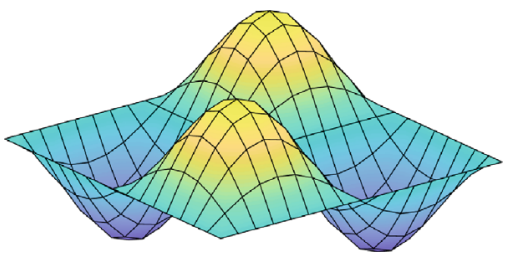

(d)

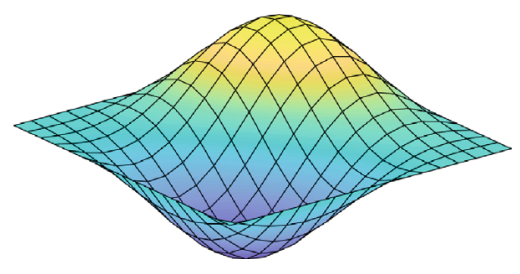

(b)

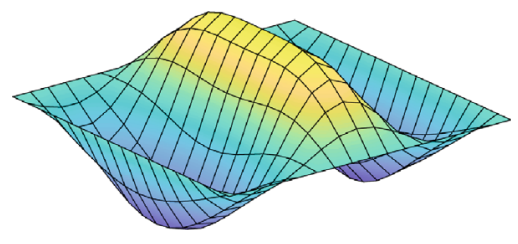

(e)

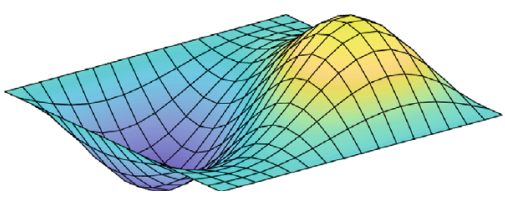

(c)

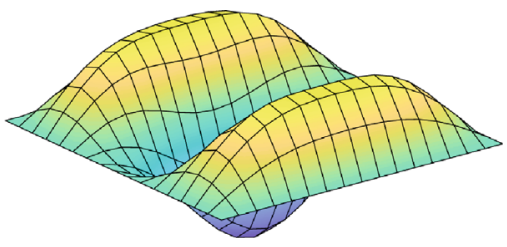

(f)

FIGURE 13: The first six mode shapes of the sandwich FG square nanoplate $\left(K_{w}=100, n=2, \mu=1, a / h=10\right.$, SSSS): (a) 1 st mode; (b) 2 nd mode; (c) 3rd mode; (d) 4th mode; (e) 5th mode; (f) 6th mode.

\section{Conclusions}

In this article, a new finite element method using the MITC4 element integrated with the nonlocal theory is proposed for the analysis of the static bending and free vibration of the sandwich functionally graded (FG) nanoplates resting on the Winkler foundation. Material properties of nanoplates are assumed to vary through thickness following two types (Type A with homogeneous core and FG material for upper and lower layers and Type B with FG material core and homogeneous materials for upper and lower layers). The obtained results of the proposed method agree excellently with others published works in the literature. The proposal method is free shear-locking and converges faster than the Q4 element and hence is very promising for the analysis of more complicated problems of the sandwich functionally graded (FG) nanoplates resting on the elastic foundation.

\section{Appendix}

\section{The Shape Function of the Quadrilateral Element}

The Lagrange interpolations in Equation (38):

$$
\begin{aligned}
& \psi_{1}=\frac{1}{4}(1-\zeta)(1-\eta) ; \psi_{2}=\frac{1}{4}(1+\zeta)(1-\eta), \\
& \psi_{3}=\frac{1}{4}(1+\zeta)(1+\eta) ; \psi_{4}=\frac{1}{4}(1-\zeta)(1+\eta)
\end{aligned}
$$

\section{Data Availability}

The data used to support the findings of this study are included within the article.

\section{Conflicts of Interest}

The authors declare that they have no conflicts of interest.

\section{Acknowledgments}

This research is funded by the Vietnam National Foundation for Science and Technology Development (NAFOSTED) under grant number 107.02-2019.330.

\section{References}

[1] F. Yang, A. C. M. Chong, D. C. C. Lam, and P. Tong, "Couple stress based strain gradient theory for elasticity," International Journal of Solids and Structures, vol. 39, no. 10, pp. 2731-2743, 2002.

[2] E. C. Aifantis, "Strain gradient interpretation of size effects," International Journal of Fracture, vol. 95, pp. 1-4, 1999.

[3] A. C. Eringen, "On differential equations of nonlocal elasticity and solutions of screw dislocation and surface waves," Journal of Applied Physics, vol. 54, no. 9, pp. 4703-4710, 1983.

[4] A. C. Eringen, Nonlocal Continuum Field Theories, Springer, New York, NY, USA, 2002.

[5] C. Li, C. W. Lim, and J. Yu, "Twisting statics and dynamics for circular elastic nanosolids by nonlocal elasticity theory," Acta Mechanica Solida Sinica, vol. 24, no. 6, pp. 484-494, 2011.

[6] R. Ansari, S. Sahmani, and B. Arash, "Nonlocal plate model for free vibrations of single-layered graphene sheets," Physis Letter $A$, vol. 375, no. 1, pp. 53-62, 2010.

[7] B. Arash and Q. Wang, "A review on the application of nonlocal elastic models in modeling of carbon nanotubes and graphenes," Computational Materials Science, vol. 51, no. 1, pp. 303-313, 2012.

[8] S. R. Asemi and A. Farajpour, "Decoupling the nonlocal elasticity equations for thermomechanical vibration of circular graphene sheets including surface effects," Physica E: Lowdimensional Systems and Nanostructures, vol. 60, pp. 80-90, 2014.

[9] S. K. Jalali, E. Jomehzadeh, and N. M. Pugno, "Influence of out-of-plane defects on vibration analysis of graphene: molecular dynamics and non-local elasticity approaches," Superlattices and Microstructures, vol. 91, pp. 331-344, 2016.

[10] R. Aghababaei and J. N. Reddy, "Nonlocal third-order shear deformation plate theory with application to bending and 
vibration of plates," Journal of Sound and Vibration, vol. 326, no. 1-2, pp. 277-289, 2009.

[11] S. C. Pradhan and T. Murmu, "Small scale effect on the buckling of single-layered graphene sheets under biaxial compression via nonlocal continuum mechanics," Computational Materials Science, vol. 47, no. 1, pp. 268-274, 2009.

[12] J. N. Reddy, "Nonlocal nonlinear formulations for bending of classical and shear deformation theories of beams and plates," International Journal of Engineering Science, vol. 48, no. 11, pp. 1507-1518, 2010.

[13] S. C. Pradhan and J. K. Phadikar, "Nonlocal theory for buckling of nano-plates," International Journal of Structural Stability and Dynamics, vol. 11, no. 3, pp. 411-429, 2011.

[14] A. Farajpour, M. Danesh, and M. Mohammadi, "Buckling analysis of variable thickness nano-plates using nonlocal continuum mechanics," Physica E, vol. 44, no. 3, pp. 719-727, 2011.

[15] T. Murmu and S. Adhikari, "Nonlocal vibration of bonded double-nano-plate-systems," Composites Part B: Engineering, vol. 42, no. 7, pp. 1901-1911, 2011.

[16] T. Aksencer and M. Aydogdu, "Forced transverse vibration of nano-plates using nonlocal elasticity," Physica E, vol. 44, no. 78, pp. 1752-1759, 2012.

[17] N. Satish, S. Narendar, and S. Gopalakrishnan, "Thermal vibration analysis of orthotropic nano-plates based on nonlocal continuum mechanics," Physica E, vol. 44, no. 9, pp. 1950-1962, 2012.

[18] Z.-B. Shen, H.-L. Tang, D. K. Li, and G. J. Tang, "Vibration of single-layered graphene sheet-based nanomechanical sensor via nonlocal Kirchhoff plate theory," Computational Materials Science, vol. 61, pp. 200-205, 2012.

[19] S. Hosseini-Hashemi, M. Zare, and R. Nazemnezhad, "An exact analytical approach for free vibration of Mindlin rectangular nano-plates via nonlocal elasticity," Composite Structures, vol. 100, pp. 290-299, 2013.

[20] S. A. Fazelzadeh and E. Ghavanloo, "Nanoscale mass sensing based on vibration of single-layered graphene sheet in thermal environments," Acta Mechanica Sinica, vol. 30, no. 1, pp. 8491, 2014

[21] S. Natarajan, S. Chakraborty, M. Thangavel, S. Bordas, and T. Rabczuk, "Size-dependent free flexural vibration behavior of functionally graded nano-plates," Computational Materials Science, vol. 65, pp. 74-80, 2012.

[22] W.-Y. Jung and S.-C. Han, "Analysis of sigmoid functionally graded material (S-FGM) nanoscale plates using the nonlocal elasticity theory," Mathematical Problems in Engineering, vol. 2013, 10 pages, 2013.

[23] M. R. Nami, M. Janghorban, and M. Damadam, "Thermal buckling analysis of functionally graded rectangular nanoplates based on nonlocal third-order shear deformation theory," Aerospace Science and Technology, vol. 41, pp. 7-15, 2015.

[24] S. Hosseini-Hashemi, M. Bedroud, and R. Nazemnezhad, "An exact analytical solution for free vibration of functionally graded circular/annular Mindlin nano-plates via nonlocal elasticity," Composite Structures, vol. 103, pp. 108-118, 2013.

[25] H. Salehipour, H. Nahvi, and A. R. Shahidi, "Exact analytical solution for free vibration of functionally graded micro/nano-plates via three-dimensional nonlocal elasticity," Physica E, vol. 66, pp. 350-358, 2015.
[26] H. Salehipour, A. R. Shahidi, and H. Nahvi, "Modified nonlocal elasticity theory for functionally graded materials," International Journal of Engineering Science, vol. 90, pp. 44-57, 2015.

[27] R. Ansari, M. Faghih Shojaei, A. Shahabodini, and M. BazdidVahdati, "Three-dimensional bending and vibration analysis of functionally graded nano-plates by a novel differential quadrature-based approach," Composite Structures, vol. 131, pp. 753-764, 2015.

[28] L. L. Ke, Y. S. Wang, J. Yang, and S. Kitipornchai, "Free vibration of size-dependent magneto-electro-elastic nanoplates based on the nonlocal theory," Acta Mechanica Sinica, vol. 30, no. 4, pp. 516-525, 2014.

[29] P. Malekzadeh, M. R. Golbahar Haghighi, and M. Shojaee, "Nonlinear free vibration of skew nano-plates with surface and small scale effects," Thin-Walled Structures, vol. 78, pp. 48-56, 2014.

[30] Z. Lee, C. Ophus, L. M. Fischer et al., "Metallic NEMS components fabricated from nanocomposite $\mathrm{Al}$ - Mo films," Nanotechnology, vol. 17, no. 12, pp. 3063-3070, 2006.

[31] M. Simsek, "Nonlocal effects in the free longitudinal vibration of axially functionally graded tapered nanorods," Computational Materials Science, vol. 61, pp. 257-265, 2012.

[32] M. Simsek and H. H. Yurtcu, "Analytical solutions for bending and buckling of functionally graded nanobeams based on the nonlocal Timoshenko beam theory," Composite Structures, vol. 97, pp. 378-386, 2013.

[33] S. Natarajan, S. Chakraborty, M. Thangavel, S. Bordas, and T. Rabczuk, "Size-dependent free flexural vibration behavior of functionally graded nanoplates," Computational Materials Science, vol. 65, pp. 74-80, 2012.

[34] R. Nazemnezhad and S. Hosseini-Hashemi, "Nonlocal nonlinear free vibration of functionally graded nanobeams," Composite Structures, vol. 110, pp. 192-199, 2014.

[35] S. Hosseini-Hashemi, I. Nahas, M. Fakher, and R. Nazemnezhad, "Surface effects on free vibration of piezoelectric functionally graded nanobeams using nonlocal elasticity," Acta Mechanica, vol. 225, no. 6, pp. 1555-1564, 2014.

[36] S. Hosseini-Hashemi, R. Nazemnezhad, and M. Bedroud, "Surface effects on nonlinear free vibration of functionally graded nanobeams using nonlocal elasticity," Applied Mathematical Modelling, vol. 38, no. 14, pp. 3538-3553, 2014.

[37] Y. Z. Wang and F. M. Li, "Static bending behaviors of nanoplate embedded in elastic matrix with small scale effects," Mechanics Research Communications, vol. 41, pp. 44-48, 2012.

[38] S. Narendar and S. Gopalakrishnan, "Nonlocal continuum mechanics based ultrasonic flexural wave dispersion characteristics of a monolayer graphene embedded in polymer matrix," Composites Part B: Engineering, vol. 43, no. 8, pp. 3096-3103, 2012.

[39] S. Pouresmaeeli, E. Ghavanloo, and S. A. Fazelzadeh, "Vibration analysis of viscoelastic orthotropic nano-plates resting on viscoelastic medium," Composite Structures, vol. 96, pp. 405-410, 2013.

[40] A. M. Zenkour and M. Sobhy, "Nonlocal elasticity theory for thermal buckling of nano-plates lying on Winkler-Pasternak elastic substrate medium," Physica E, vol. 53, pp. 251-259, 2013.

[41] M. Panyatong, B. Chinnaboon, and S. Chucheepsakul, "Incorporated effects of surface stress and nonlocal elasticity on bending analysis of nano-plates embedded in an elastic 
medium," Suranaree Journal of Science and Technology, vol. 22, no. 1, pp. 21-33, 2015.

[42] O. C. Zienkiewicz, R. L. Taylor, and J. M. Too, "Reduced integration technique in general analysis of plates and shells," International Journal for Numerical Methods in Engineering, vol. 3, no. 2, pp. 275-290, 1971.

[43] K.-U. Bletzinger, M. Bischoff, and E. Ramm, "A unified approach for shear-locking-free triangular and rectangular shell finite elements," Computers \& Structures, vol. 75, no. 3, pp. 321-334, 2000.

[44] A. Tessler and T. J. R. Hughes, "A three-node Mindlin plate element with improved transverse shear," Computer Methods in Applied Mechanics and Engineering, vol. 50, no. 1, pp. 71101, 1985.

[45] E. N. Dvorkin and K. J. Bathe, "A continuum mechanics based four-node shell element for general non-linear analysis," Engineering Computations, vol. 1, no. 1, pp. 77-88, 1984.

[46] E. N. Dvorkin, D. Pantuso, and E. A. Repetto, "A formulation of the MITC4 shell element for finite strain elasto-plastic analysis," Computer Methods in Applied Mechanics and Engineering, vol. 125, no. 1-4, pp. 17-40, 1995.

[47] P.-S. Lee and K.-J. Bathe, "Development of MITC isotropic triangular shell finite elements," Computers and Structures, vol. 82, no. 11-12, pp. 945-962, 2004.

[48] K. J. Bathe and E. N. Dvorkin, "A formulation of general shell elements - the use of mixed interpolation of tensorial components," International Journal for Numerical Methods in Engineering, vol. 22, no. 3, pp. 697-722, 1986.

[49] J. N. Reddy, Mechanics of Laminated Composite Plate and Shell, CRC Press, Boca Raton, FL, USA, 2nd edition, 2004.

[50] H. T. Thai and D. H. Choi, "A refined plate theory for functionally graded plates resting on elastic foundation," Composites Science and Technology, vol. 71, no. 16, pp. 1850-1858, 2011.

[51] H. T. Thai, T. Park, and D. H. Choi, "An efficient shear deformation theory for vibration of functionally graded plates," Archive of Applied Mechanics, vol. 83, no. 1, pp. 137149, 2013.

[52] M. Sobhy, "A comprehensive study on FGM nano-plates embedded in an elastic medium," Composite Structures, vol. 134, pp. 966-980, 2015. 